Louisiana State University

LSU Digital Commons

$1-21-2004$

\title{
Detector description and performance for the first coincidence observations between LIGO and GEO
}

\author{
B. Abbott \\ California Institute of Technology
}

R. Abbott

LIGO Livingston

R. Adhikari

Massachusetts Institute of Technology

\author{
A. Ageev \\ Lomonosov Moscow State University \\ B. Allen \\ University of Wisconsin-Milwaukee
}

See next page for additional authors

Follow this and additional works at: https://digitalcommons.Isu.edu/physics_astronomy_pubs

\section{Recommended Citation}

Abbott, B., Abbott, R., Adhikari, R., Ageev, A., Allen, B., Amin, R., Anderson, S., Anderson, W., Araya, M., Armandula, H., Asiri, F., Aufmuth, P., Aulbert, C., Babak, S., Balasubramanian, R., Ballmer, S., Barish, B., Barker, D., Barker-Patton, C., Barnes, M., Barr, B., Barton, M., Bayer, K., Beausoleil, R., Belczynski, K., Bennett, R., Berukoff, S., Betzwieser, J., Bhawal, B., Bilenko, I., Billingsley, G., Black, E., \& Blackburn, K. (2004). Detector description and performance for the first coincidence observations between LIGO and GEO. Nuclear Instruments and Methods in Physics Research, Section A: Accelerators, Spectrometers, Detectors and Associated Equipment, 517 (1-3), 154-179. https://doi.org/10.1016/j.nima.2003.11.124

This Article is brought to you for free and open access by the Department of Physics \& Astronomy at LSU Digital Commons. It has been accepted for inclusion in Faculty Publications by an authorized administrator of LSU Digital Commons. For more information, please contact ir@lsu.edu. 


\section{Authors}

B. Abbott, R. Abbott, R. Adhikari, A. Ageev, B. Allen, R. Amin, S. B. Anderson, W. G. Anderson, M. Araya, H. Armandula, F. Asiri, P. Aufmuth, C. Aulbert, S. Babak, R. Balasubramanian, S. Ballmer, B. C. Barish, D.

Barker, C. Barker-Patton, M. Barnes, B. Barr, M. A. Barton, K. Bayer, R. Beausoleil, K. Belczynski, R. Bennett, S. J. Berukoff, J. Betzwieser, B. Bhawal, I. A. Bilenko, G. Billingsley, E. Black, and K. Blackburn 


\section{Detector description and performance for the first coincidence observations between LIGO and GEO}

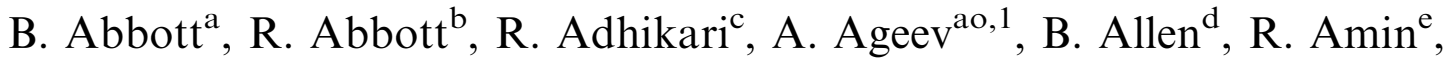 S.B. Anderson ${ }^{a}$, W.G. Anderson ${ }^{f}$, M. Araya ${ }^{a}$, H. Armandula ${ }^{a}$, F. Asiri ${ }^{a}, 2$, P. Aufmuth ${ }^{\mathrm{g}}$, C. Aulbert ${ }^{\mathrm{h}}$, S. Babak ${ }^{\mathrm{i}}$, R. Balasubramanian ${ }^{\mathrm{i}}, \mathrm{S}$. Ballmer ${ }^{\mathrm{c}}$, B.C. Barish ${ }^{\mathrm{a}}$, D. Barker ${ }^{\mathrm{i}}$, C. Barker-Patton ${ }^{\mathrm{j}}$, M. Barnes ${ }^{\mathrm{a}}$, B. Barr ${ }^{\mathrm{k}}$, M.A. Barton ${ }^{\mathrm{a}}$, K. Bayer ${ }^{\mathrm{c}}$, R. Beausoleil ${ }^{1,3}$, K. Belczynski ${ }^{\mathrm{m}}$, R. Bennett ${ }^{\mathrm{k}, 4}$, S.J. Berukoff ${ }^{\mathrm{h}, 5}$, J. Betzwieser ${ }^{\mathrm{c}}$, B. Bhawal ${ }^{\mathrm{a}}$, I.A. Bilenko ${ }^{\text {ao }}$, G. Billingsley ${ }^{\mathrm{a}}$, E. Black $^{\mathrm{a}}$, K. Blackburn ${ }^{\mathrm{a}}$, B. Bland-Weaver, B. Bochner ${ }^{\mathrm{c}, 6}$, L. Bogue ${ }^{\mathrm{a}}$, R. Bork ${ }^{\mathrm{a}}$, S. Bose $^{\mathrm{n}}$,

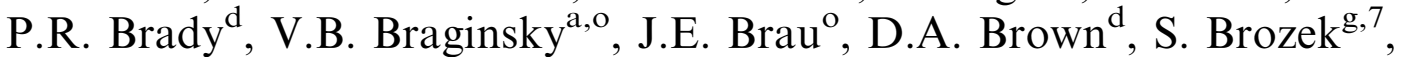

A. Bullington ${ }^{1}$ A. Buonanno ${ }^{\text {p, } 8}$, R. Burgess ${ }^{c}$, D. Busby ${ }^{\mathrm{a}}$, W.E. Butler ${ }^{\mathrm{q}}$, R.L. Byer ${ }^{1}$, L. Cadonatic, G. Cagnolik, J.B. Camp ${ }^{\mathrm{r}}$, C.A. Cantley ${ }^{\mathrm{k}}$, L. Cardenas ${ }^{\mathrm{a}}$, K. Carter ${ }^{\mathrm{b}}$, M.M. Casey ${ }^{\mathrm{k}}$, J. Castiglione ${ }^{\mathrm{e}}$, A. Chandler ${ }^{\mathrm{a}}$, J. Chapsky ${ }^{\mathrm{a}, 9}$, P. Charlton ${ }^{\mathrm{a}}$, S. Chatterjic ${ }^{\mathrm{c}}$, Y. Chen ${ }^{\mathrm{p}}$, V. Chickarmane ${ }^{\mathrm{s}}$, D. Chin ${ }^{\mathrm{t}}$, N. Christensen ${ }^{\mathrm{u}}$, D. Churches ${ }^{\mathrm{i}}$,

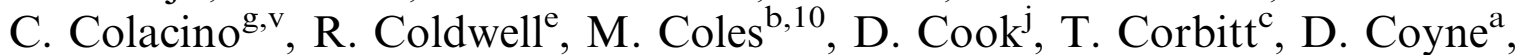
J.D.E. Creighton ${ }^{\mathrm{d}}$, T.D. Creighton ${ }^{\mathrm{a}}$, D.R.M. Crooks ${ }^{\mathrm{k}}$, P. Csatorday ${ }^{\mathrm{c}}$, B.J. Cusack ${ }^{\mathrm{w}}$, C. Cutler ${ }^{\mathrm{h}}$, E. D'Ambrosio ${ }^{\mathrm{a}}$, K. Danzmann ${ }^{\mathrm{g}, \mathrm{v}, \mathrm{x}}$, R. Davies ${ }^{\mathrm{i}}$,

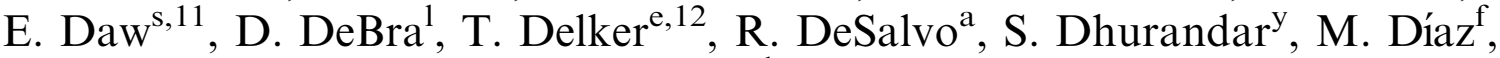
H. Ding ${ }^{\mathrm{a}}$, R.W.P. Drever ${ }^{\mathrm{z}}$, R.J. Dupuis ${ }^{\mathrm{k}}$, C. Ebeling ${ }^{\mathrm{u}}$, J. Edlund ${ }^{\mathrm{a}}$, P. Ehrens ${ }^{\mathrm{a}}$, E.J. Elliffe ${ }^{\mathrm{k}}$, T. Etzel ${ }^{\mathrm{a}}$, M. Evans ${ }^{\mathrm{a}}$, T. Evans ${ }^{\mathrm{b}}$, C. Fallnich ${ }^{\mathrm{g}}$, D. Farnham ${ }^{\mathrm{a}}$,

\footnotetext{
*Corresponding author. MIT NW17-161, 175 Albany Str., Cambridge, MA 02139, USA Tel.: + 1-617-253-6411; fax: + 1-617-2537014.

E-mail addresses: dhs@ligo.mit.edu (D.H. Shoemaker).

${ }^{1}$ Currently at Syracuse University.

${ }^{2}$ Currently at Stanford Linear Accelerator Center.

${ }^{3}$ Currently at HP Laboratories.

${ }^{4}$ Currently at Rutherford Appleton Laboratory.

${ }^{5}$ Currently at University of California, Los Angeles.

${ }^{6}$ Currently at Hofstra University.

${ }^{7}$ Currently at Siemens AG.

${ }^{8}$ Permanent address: GReCO, Institut d'Astrophysique de Paris (CNRS).

${ }^{9}$ Currently at NASA Jet Propulsion Laboratory.

${ }^{10}$ Currently at National Science Foundation.

${ }^{11}$ Currently at University of Sheffield.

${ }^{12}$ Currently at Ball Aerospace Corporation.
} 
M.M. Fejer ${ }^{1}$, M. Fine ${ }^{\mathrm{a}}$, L.S. Finn ${ }^{\mathrm{aa}}$, É. Flanagan ${ }^{\mathrm{ab}}$, A. Freise $^{\mathrm{v}, 13}$, R. Frey $^{\mathrm{o}}$, P. Fritschel ${ }^{\mathrm{c}}$, V. Frolov ${ }^{\mathrm{b}}$, M. Fyffe ${ }^{\mathrm{b}}$, K.S. Ganezer ${ }^{\text {ac }}$, J.A. Giaime ${ }^{\mathrm{s}}$, A. Gillespie ${ }^{\mathrm{a}}$,14, K. Goda ${ }^{c}$, G. González , S. Goßler ${ }^{\mathrm{g}}$, P. Grandclément ${ }^{\mathrm{m}}$, A. Grant ${ }^{\mathrm{k}}$, C. Gray ${ }^{\mathrm{j}}$, A.M. Gretarsson ${ }^{\mathrm{b}}$, D. Grimmett ${ }^{\mathrm{a}}$, H. Grote ${ }^{\mathrm{v}}$, S. Grunewald ${ }^{\mathrm{h}}$, M. Guenther ${ }^{\mathrm{j}}$, E. Gustafson ${ }^{1,15}$, R. Gustafson ${ }^{\text {t, W.O. Hamilton }}{ }^{\mathrm{s}}$, M. Hammond ${ }^{\mathrm{b}}$, J. Hanson ${ }^{\mathrm{b}}$, C. Hardham ${ }^{1}$, G. Harry ${ }^{\mathrm{c}}$, A. Hartunian ${ }^{\mathrm{a}}$, J. Heefner ${ }^{\mathrm{a}}$, Y. Hefetz ${ }^{\mathrm{c}}$, G. Heinzel ${ }^{\mathrm{v}}$, I.S. Heng ${ }^{\mathrm{g}}$, M. Hennessy ${ }^{1}$, N. Hepler ${ }^{\mathrm{v}}$, A. Heptonstall ${ }^{\mathrm{k}}$, M. Heurs ${ }^{\mathrm{g}}$, M. Hewitson ${ }^{\mathrm{k}}$, N. Hindman ${ }^{\mathrm{j}}$, P. Hoang ${ }^{\mathrm{a}}$, J. Hough ${ }^{\mathrm{k}}$, M. Hrynevych ${ }^{\mathrm{a}, 16}$, W. Hua ${ }^{1}$, R. Ingley ${ }^{\text {ad }}$, M. Ito ${ }^{o}$, Y. Itoh ${ }^{\mathrm{h}}$, A. Ivanov ${ }^{\mathrm{a}}$, O. Jennrich ${ }^{\mathrm{k}, 17}$, W.W. Johnson ${ }^{\mathrm{s}}$, W. Johnston ${ }^{\mathrm{f}}$, L. Jones $^{\mathrm{a}}$, D. Jungwirth ${ }^{\mathrm{a}, 18}$, V. Kalogera ${ }^{\mathrm{m}}$, E. Katsavounidis ${ }^{\mathrm{c}}$, K. Kawabe ${ }^{\mathrm{x}, \mathrm{v}}$, S. Kawamura ${ }^{\text {ae }}$, W. Kells ${ }^{\mathrm{a}}$, J. Kern ${ }^{\mathrm{b}}$, A. Khan ${ }^{\mathrm{b}}$, S. Killbourn ${ }^{\mathrm{k}}$, C.J. Killow ${ }^{\mathrm{k}}$, C. Kim ${ }^{\mathrm{m}}$, C. King ${ }^{\mathrm{a}}$, P. King ${ }^{\mathrm{a}}$, S. Klimenko ${ }^{\mathrm{e}}$, P. Kloevekorn ${ }^{\mathrm{v}}$, S. Koranda ${ }^{\mathrm{d}}$, K. Kötter ${ }^{\text {g }}$, J. Kovalik ${ }^{\mathrm{b}}$, D. Kozak ${ }^{\mathrm{a}}$, B. Krishnan ${ }^{\mathrm{h}}$, M. Landry ${ }^{\mathrm{j}}$, J. Langdale ${ }^{\mathrm{b}}$, B. Lantz, R. Lawrence ${ }^{\mathrm{c}}$, A. Lazzarini ${ }^{\mathrm{a}}$, M. Lei ${ }^{\mathrm{a}}$, V. Leonhardt ${ }^{\mathrm{g}}$, I. Leonor ${ }^{\mathrm{o}}$, K. Libbrecht ${ }^{\mathrm{a}}$, P. Lindquist ${ }^{\mathrm{a}}, \mathrm{S}$. Liu ${ }^{\mathrm{a}}$, J. Logan $^{\mathrm{a}, 19}$, M. Lormand ${ }^{\mathrm{b}}$, M. Lubinski $^{\mathrm{j}}$, H. Lück ${ }^{\mathrm{g}, \mathrm{v}}$, T.T. Lyons ${ }^{\mathrm{a}, 18}$, B. Machenschalk ${ }^{\mathrm{h}}$, M. MacInnis ${ }^{\mathrm{c}}$, M. Mageswaran ${ }^{\mathrm{a}}$, K. Mailand ${ }^{\mathrm{a}}$, W. Majid ${ }^{\mathrm{a}, 8}$, M. Malec ${ }^{\mathrm{g}}$, F. Mann ${ }^{\mathrm{a}}$, A. Marin ${ }^{\mathrm{c}, 20}$, S. Márka ${ }^{\mathrm{a}}$,

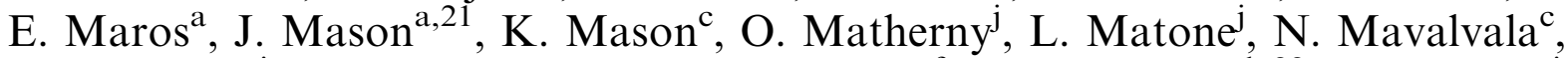
R. McCarthy ${ }^{\mathrm{j}}$, D.E. McClelland ${ }^{\mathrm{w}}$, M. McHugh ${ }^{\text {af }}$, P. McNamara ${ }^{\mathrm{k}, 22}$, G. Mendelli, S. Meshkov ${ }^{\text {a }}$, C. Messenger ${ }^{\text {ad }}$, V.P. Mitrofanov ${ }^{\text {ao }}$, G. Mitselmakher ${ }^{\mathrm{e}}$, R. Mittleman ${ }^{c}$, O. Miyakawa ${ }^{a}$, S. Miyoki ${ }^{\mathrm{a}, 23}$, S. Mohanty ${ }^{\mathrm{h}}$, G. Moreno, K. Mossavi ${ }^{\mathrm{v}}$, B. Mours ${ }^{\mathrm{a}, 24}$, G. Mueller ${ }^{\mathrm{e}}$, S. Mukherjee ${ }^{\mathrm{h}}$, J. Myers ${ }^{\mathrm{j}}$, S. Nagano ${ }^{\mathrm{v}}$,

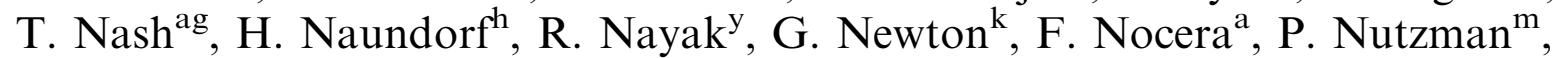
T. Olson ${ }^{\text {ah }}$, B. O'Reilly ${ }^{b}$, D. J. Ottaway ${ }^{\mathrm{c}}$, A. Ottewill ${ }^{\mathrm{d}, 25}$, D. Ouimette ${ }^{\mathrm{a}, 17}$, H. Overmier ${ }^{\text {b }}$, B.J. Owen ${ }^{\text {aa }}$, M.A. Papa ${ }^{\text {h }}$, C. Parameswariah ${ }^{b}$, V. Parameswariah ${ }^{\mathrm{j}}$,

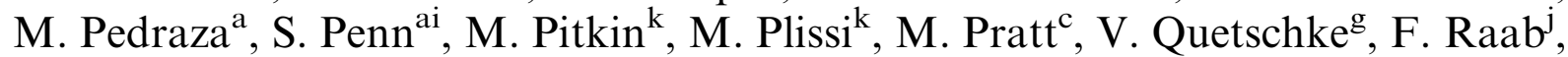

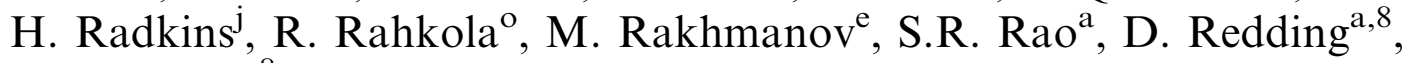
M.W. Regehr ${ }^{a, 8}$, T. Regimbau ${ }^{\text {c }}$, K.T. Reilly ${ }^{\mathrm{a}}$, K. Reithmaier ${ }^{\mathrm{a}}$, D.H. Reitze ${ }^{\mathrm{e}}$,

\footnotetext{
${ }^{13}$ Currently at European Gravitational Observatory.

${ }^{14}$ Currently at Intel Corp.

${ }^{15}$ Currently at Lightconnect Inc.

${ }^{16}$ Currently at Keck Observatory.

${ }^{17}$ Currently at ESA Science and Technology Center.

${ }^{18}$ Currently at Raytheon Corporation.

${ }^{19}$ Currently at Mission Research Corporation.

${ }^{20}$ Currently at Harvard University.

${ }^{21}$ Currently at Lockheed-Martin Corporation.

${ }^{22}$ Currently at NASA Goddard Space Flight Center.

${ }^{23}$ Permanent address: University of Tokyo, Institute for Cosmic Ray Research.

${ }^{24}$ Currently at Laboratoire d'Annecy-le-Vieux de Physique des Particules.

${ }^{25}$ Permanent address: University College Dublin.
} 
S. Richman ${ }^{\mathrm{c}, 26}$, R. Riesen ${ }^{\mathrm{b}}$, K. Riles ${ }^{\mathrm{t}}$, A. Rizzi ${ }^{\mathrm{b}, 27}$, D.I. Robertson ${ }^{\mathrm{k}}$, N.A. Robertson ${ }^{\mathrm{k}, 1}$, L. Robison ${ }^{\mathrm{a}}$, S. Roddy ${ }^{\mathrm{b}}$, J. Rollins ${ }^{\mathrm{c}}$, J.D. Romano ${ }^{\mathrm{f}}$, J. Romie ${ }^{\mathrm{a}}$, H. Rong ${ }^{\mathrm{e}, 13}$, D. Rose ${ }^{\mathrm{a}}$, E. Rotthoff ${ }^{\mathrm{aa}}$, S. Rowan ${ }^{\mathrm{k}}$, A. Rüdiger ${ }^{\mathrm{v}, \mathrm{x}}$, P. Russell ${ }^{\mathrm{a}}$, K. Ryan ${ }^{j}$, I. Salzman ${ }^{\text {a }}$, G.H. Sanders ${ }^{\mathrm{a}}$, V. Sannibale ${ }^{\mathrm{a}}$, B. Sathyaprakash ${ }^{\mathrm{i}}$, P.R. Saulson ${ }^{\text {aj }}$, R. Savage ${ }^{\mathrm{j}}$, A. Sazonov ${ }^{\mathrm{e}}$, R. Schilling ${ }^{\mathrm{v}, \mathrm{x}}$, K. Schlaufman ${ }^{\mathrm{aa}}$, V. Schmidt ${ }^{\mathrm{a}, 28}$, R. Schofield ${ }^{\mathrm{o}}$, M. Schrempel ${ }^{\mathrm{g}, 29}$, B.F. Schutz ${ }^{\mathrm{h}, \mathrm{i}}$, P. Schwinberg, S.M. Scott ${ }^{\mathrm{w}}$, A.C. Searle ${ }^{\mathrm{w}}$, B. Sears ${ }^{\mathrm{a}}$, S. Seel ${ }^{\mathrm{a}}$, A.S. Sengupta ${ }^{\mathrm{y}}$, C.A. Shapiro ${ }^{\mathrm{a} a, 30}$, P. Shawhan ${ }^{\mathrm{a}}$, D.H. Shoemaker ${ }^{\mathrm{c}, *}$, Q.Z. Shu ${ }^{\mathrm{e}, 31}$, A. Sibley ${ }^{\mathrm{b}}$, X. Siemens ${ }^{\mathrm{d}}$, L. Sievers ${ }^{\mathrm{a}, 8}$, D. Sigg ${ }^{\mathrm{j}}$, A.M. Sintes ${ }^{\mathrm{h}, \mathrm{ak}}$, K. Skeldon ${ }^{\mathrm{k}}$, J.R. Smith ${ }^{\mathrm{v}}$, M. Smith ${ }^{\mathrm{c}}$, M.R. Smith ${ }^{\mathrm{a}}$, P. Sneddon ${ }^{\mathrm{k}}$, R. Spero ${ }^{\mathrm{a}, 8}$, G. Stapfer ${ }^{\mathrm{b}}$, K.A. Strain ${ }^{\mathrm{k}}$, D. Strom ${ }^{\mathrm{o}}$, A. Stuver ${ }^{\text {aa }}$, T. Summerscales ${ }^{\text {aa }}$, M.C. Sumner ${ }^{\text {a }}$, P.J. Sutton ${ }^{\text {aa }}$, J. Sylvestre ${ }^{a}$, A. Takamori ${ }^{\mathrm{a}}$, D.B. Tanner ${ }^{\mathrm{e}}$, H. Tariq ${ }^{\mathrm{a}}$, I. Taylor ${ }^{\mathrm{i}}$, R. Taylor ${ }^{\mathrm{a}}$, K.S. Thorne ${ }^{\mathrm{p}}$,

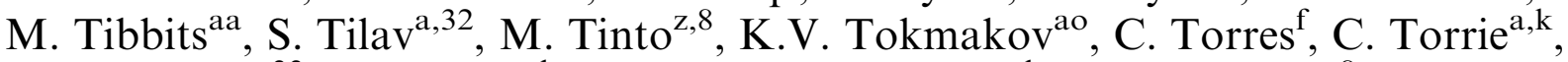

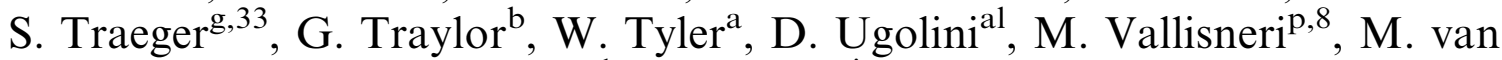
Putten $^{\text {c }}$, S. Vass ${ }^{\mathrm{a}}$, A. Vecchio ${ }^{\text {ad }}$, C. Vorvick ${ }^{\mathrm{j}}$, S.P. Vyatchanin ${ }^{\mathrm{ao}}$, L. Wallace , $^{\mathrm{a}}$, H. Walther ${ }^{\mathrm{x}}$, H. Ward ${ }^{\mathrm{k}}$, B. Ware ${ }^{\mathrm{a}, 8}, \mathrm{~K}$. Watts ${ }^{\mathrm{b}}$, D. Webber ${ }^{\mathrm{a}}$, A. Weidner ${ }^{\mathrm{v}, \mathrm{x}}$, U. Weiland ${ }^{\mathrm{g}}$, A. Weinstein ${ }^{\mathrm{a}}$, R. Weiss ${ }^{\mathrm{c}}$, H. Welling ${ }^{\mathrm{g}}$, L. Wen ${ }^{\mathrm{a}}$, S. Wen ${ }^{\mathrm{s}}$, J.T. Whelan $^{\text {af }}$, S.E. Whitcomb ${ }^{\text {a }}$, B.F. Whiting ${ }^{\mathrm{e}}$, P.A. Willems ${ }^{\mathrm{a}}$, P.R. Williams ${ }^{\mathrm{h}, 34}$, R. Williams ${ }^{\mathrm{z}}$, B. Willke ${ }^{\mathrm{g}, \mathrm{v}}$, A. Wilson ${ }^{\mathrm{a}}$, B.J. Winjum ${ }^{\mathrm{aa}, 4}, \mathrm{~W}$. Winkler ${ }^{\mathrm{v}, \mathrm{x}}, \mathrm{S}_{\text {. Wise }}$, $^{\mathrm{e}}$ A.G. Wiseman ${ }^{\mathrm{d}}$, G. Woan ${ }^{\mathrm{k}}$, R. Wooley ${ }^{\mathrm{b}}$, J. Worden $^{\mathrm{j}}$, I. Yakushin $^{\mathrm{b}}$, H. Yamamoto ${ }^{\text {a }}$ S. Yoshida ${ }^{\text {am }}$, I. Zawischa ${ }^{\mathrm{g}, 35}$, L. Zhang ${ }^{\mathrm{a}}$, N. Zotov ${ }^{\text {an }}$, M. Zucker ${ }^{\mathrm{b}}$, J. Zweizig ${ }^{\mathrm{a}}$

\author{
${ }^{a}$ LIGO_California Institute of Technology, Pasadena, CA 91125, USA \\ ${ }^{\mathrm{b}}$ LIGO Livingston Observatory, Livingston, LA 70754, USA \\ ${ }^{\mathrm{c}}$ LIGO_Massachusetts Institute of Technology, Cambridge, MA 02139, USA \\ ${ }^{\mathrm{d}}$ University of Wisconsin-Milwaukee, Milwaukee, WI 53201, USA \\ ${ }^{\mathrm{e}}$ University of Florida, Gainsville, FL 32611, USA \\ ${ }^{\mathrm{f}}$ The University of Texas at Brownsville and Texas Southmost College, Brownsville, TX 78520, USA \\ ${ }^{\mathrm{g}}$ Universität Hannover, D-30167 Hannover, Germany \\ ${ }^{\mathrm{h}}$ Albert-Einstein-Institut, Max-Planck-Institut für Gravitationsphysik, D-14476 Golm, Germany \\ ${ }^{\mathrm{i}}$ Cardiff University, Cardiff CF2 $3 Y B, U K$ \\ ${ }^{\mathrm{j}}$ LIGO Hanford Observatory, Richland WA 99352, USA \\ ${ }^{\mathrm{k}}$ University of Glasgow, Glasgow G12 8QQ, UK \\ ${ }^{1}$ Stanford University, Stanford, CA 94305, USA
}

\footnotetext{
${ }^{26}$ Currently at Research Electro-Optics Inc.

${ }^{27}$ Currently at Institute of Advanced Physics, Baton Rouge, LA.

${ }^{28}$ Currently at European Commission, DG Research, Brussels, Belgium.

${ }^{29}$ Currently at Spectra Physics Corporation.

${ }^{30}$ Currently at University of Chicago.

${ }^{31}$ Currently at LightBit Corporation.

${ }^{32}$ Currently at University of Delaware.

${ }^{33}$ Currently at Carl Zeiss GmbH.

${ }^{34}$ Currently at Shanghai Astronomical Observatory.

${ }^{35}$ Currently at Laser Zentrum Hannover.
} 


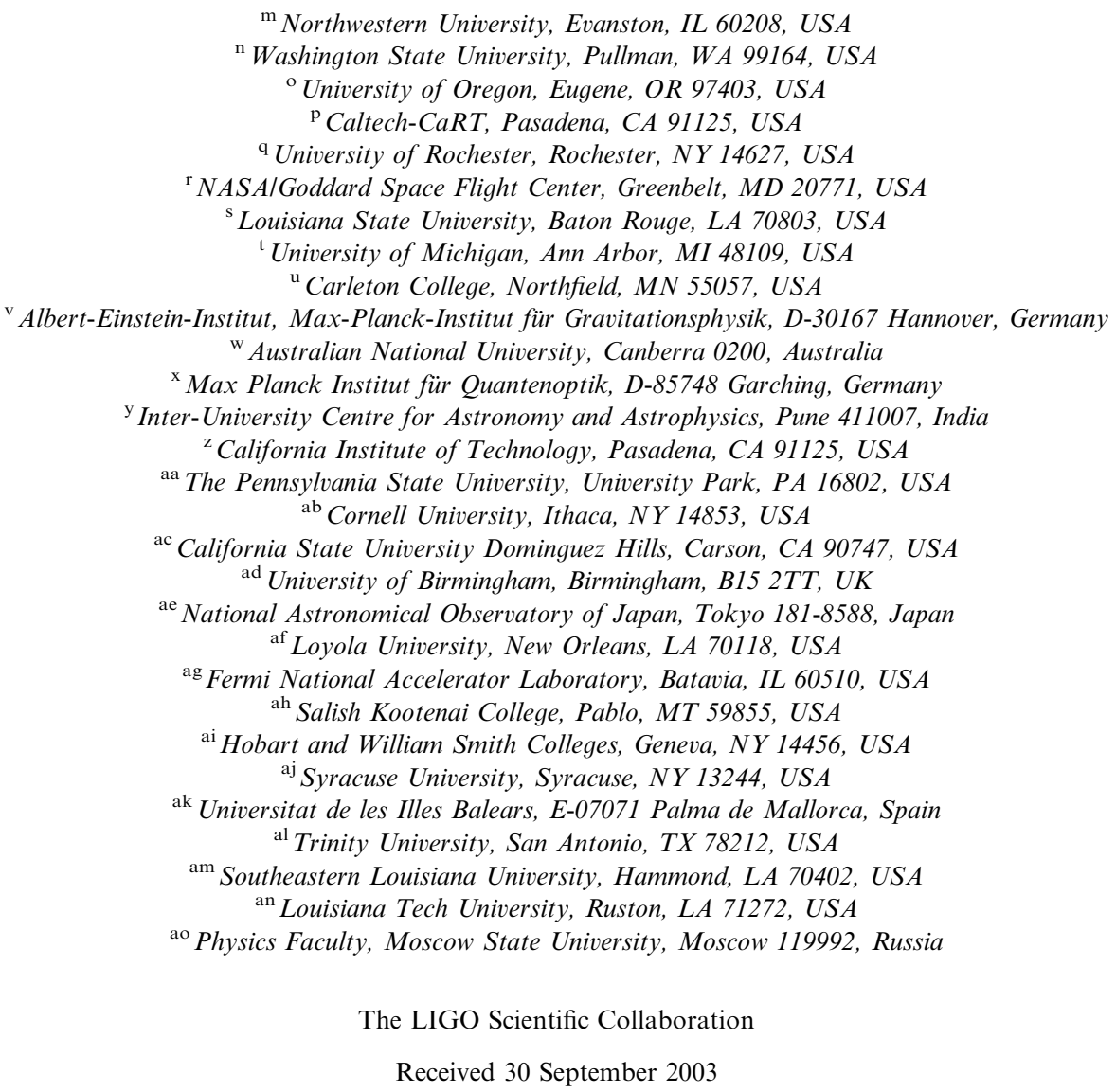

\begin{abstract}
For 17 days in August and September 2002, the LIGO and GEO interferometer gravitational wave detectors were operated in coincidence to produce their first data for scientific analysis. Although the detectors were still far from their design sensitivity levels, the data can be used to place better upper limits on the flux of gravitational waves incident on the earth than previous direct measurements. This paper describes the instruments and the data in some detail, as a companion to analysis papers based on the first data.
\end{abstract}

(C) 2003 Elsevier B.V. All rights reserved.

PACS: $04.89 . \mathrm{Nn} ;$ 07.60.Ly; 95.45. + i; 95.55.Ym

Keywords: LIGO; Gravitational wave; Interferometer; Observatory

\section{Introduction}

A number of laboratories around the world (TAMA [1], VIRGO [2], GEO [3,4], LIGO [5,6]) are developing detectors for gravitational waves using laser interferometers to sense the very small strains anticipated from astrophysical sources. In a joint effort, two of these laboratories, LIGO and GEO 600, have performed their first scientific observations. This note is intended to provide greater detail in the description of the detectors themselves as a companion to papers describing 
the data analysis and astrophysical conclusions from this Science Run (designated S1).

Both GEO 600 and LIGO use the principle of the Michelson interferometer, with its high sensitivity to differential changes $\Delta L=L_{1}-L_{2}$ of the lengths $L$ in the two perpendicular arm lengths $L_{1}$ and $L_{2}$, to detect strains of the order of $h=$ $\Delta L / L=10^{-20}$ over a wide frequency range. The required sensitivity of the interferometric readout is achieved through the use of high circulating laser power (to improve the shot-noise limited fringe resolution) and through techniques to store the light in the interferometer arms (to increase the phase shift due to a passing gravitational wave). The frequency range of interest for these instruments lies in the audio band $(\sim 50-5000 \mathrm{~Hz})$, leading to gravitational wavelengths $\lambda_{\mathrm{gw}}=c / f_{\mathrm{gw}}$ of hundreds of $\mathrm{km}$. Because practical groundbased detectors are short compared to the wavelength, long interferometer arms are chosen to increase the sensitivity of the instrument. A vacuum system protects the beams from variations in the light path due to air density fluctuations. The test masses, which also serve as mirrors for the Michelson interferometer, are suspended as pendulums and respond as free masses above their $\sim 1 \mathrm{~Hz}$ resonant frequency. External mechanical disturbances are suppressed through seismic isolation systems, and the in-band intrinsic thermal noise is controlled via careful choice of materials and construction techniques.

\section{1. $L I G O$}

The LIGO Observatory construction started in 1994 at the LIGO site in Hanford, Washington, USA. Construction at the Livingston, Louisiana, USA site began a year later in June 1995. The buildings and $4 \mathrm{~km}$ concrete arm-support foundations were completed in 1998. The vacuum systems were completed in 1999, and detector installation was substantially completed in 2000 . The first operation of a LIGO interferometer took place in October 2000. This marked the initiation of the commissioning, consisting of periods of intense testing and tuning of subsystems, separated by periods where the interferometers were run as complete systems. These engineering runs were primarily intended to assess the progress toward full detector operation. However, they were also used to collect data that could be used to test data handling, archiving and analysis software. Progress through the commissioning phase has been steady, both in terms of improving sensitivity and in terms of reliable operation with a reasonable duty cycle. By summer 2002, the improvements had been sufficient that a short duration Science Run could be expected to achieve limits on the observations of gravitational waves that would be comparable to or better than previous experimental limits. Consequently, a two-week observation period was scheduled, and other laboratories operating interferometer detectors were invited to join in simultaneous operation, as documented here. Further progress in sensitivity has subsequently been achieved through additional commissioning.

\subsection{GEO 600}

The construction of GEO 600 started in 1995 as a German/British collaboration on a site near Hannover, Germany. Because the site constrained the length of the arms to $600 \mathrm{~m}$, an advanced optical layout and novel techniques for the suspension systems were included in the detector design. After the buildings and the trenches were finished in 1997 the complete vacuum system was installed and tested. The construction phase was followed by the installation of the two suspended triangular mode cleaners which have been operating reliably since 2000 . To gain experience with the alignment and length control of long baseline cavities the commissioning continued with the installation of a $1200 \mathrm{~m}$ long Fabry-Perot cavity formed by one interferometer arm and the power recycling mirrors. To reduce the risk of contaminating or damaging the expensive main interferometer mirrors, lower grade test mirrors suspended in steel wire slings were used for the $1200 \mathrm{~m}$ cavity experiment and for the commissioning of the power recycled Michelson interferometer which started in summer 2001. A first engineering test run was conducted in Jan 2002 in coincidence with a LIGO engineering run. 
The installation of the automatic alignment system for the Michelson interferometer and for the power-recycling cavity was a key step towards a duty cycle of more than $98 \%$ which was achieved in the 17 day S1 Science Run. The instrument ran as a power-recycled folded-arm Michelson for S1; commissioning of signal recycling started after S1 and is expected to bring the GEO detector a significant step closer to its design sensitivity.

\section{Purpose of the S1 science run}

The primary goal of the S1 run was to collect a significant body of data to analyze for gravitational waves. Although the sensitivity of all the instruments was far from the design goal and the relatively short run time made it unlikely that a positive detection would be made, it was expected that upper limits could be derived from the data that would be comparable to or better than previous gravitational wave observations. Furthermore, the analysis provided the opportunity to test the methodologies with real gravitational wave detector data. Estimates of sensitivity for gravitational wave interferometers have almost always been based on the assumption of Gaussian noise. While this is a good point of departure for many of the limiting noise sources (e.g., shot noise or thermal noise), many others (e.g., seismic noise) are not expected to be so well-behaved. Thus, letting the data analysis confront the behavior of real noise as early as possible is crucial to developing and testing the analysis techniques.

Other goals for S1 were aimed at improving our understanding of the detectors and their operation. These include:

(1) Investigating the factors that influence duty cycle for the interferometers. Long periods of operation with stable conditions are important for understanding the causes for the interferometers to lose 'lock' (loss of resonance condition for light in the interferometer cavities and consequent loss of linear operation of the sensing system)

(2) Characterization of drifts in alignment and optimization of the alignment control systems
(3) Testing and optimization of on-line monitoring tools for assessing performance and maintaining high sensitivity

(4) Training and practice for instrument operators and scientific monitors.

This paper provides a description of the LIGO and GEO interferometers as they were used in the S1 run. ${ }^{36}$ It is intended as a companion to the data analysis papers based on data from this run. Because commissioning was still underway, many parts of the detectors were not in their intended final operational configuration, and an important emphasis of this paper will be to identify and highlight those differences.

\section{The LIGO detector array}

The LIGO detector array comprises three interferometers at two sites. The LIGO Livingston Observatory (LLO) contains three main instrument bays at the vertex and ends of the L-shaped site and houses a single interferometer with $4 \mathrm{~km}$ long arms (designated L1). The LIGO Hanford Observatory (LHO) has two additional experimental halls at the midpoint in each arm which enable it to accommodate two interferometers, one with $4 \mathrm{~km}$ long arms (designated $\mathrm{H} 1$ ) and one with $2 \mathrm{~km}$ arms (H2). The orientation of the Hanford site was chosen to be as closely aligned (modulo $90^{\circ}$ ) to the Livingston site as possible, consistent with the earth's curvature and the need for the sites to be level; this maximizes the common response to a signal, given the quadrupolar form of the anticipated gravitational waves. The arms have an included angle of $90.000^{\circ}$. The locations and orientations of the two LIGO sites are given in Table 1.

The observatories have a support infrastructure of clean rooms, preparation laboratories, maintenance shops, and computer networking for control, data acquisition and analysis, as well as offices for site staff and meeting spaces for larger gatherings. The vacuum system can be divided into

\footnotetext{
${ }^{36} \mathrm{~A}$ shorter period of simultaneous observations between TAMA, GEO, and LIGO also took place during the period of this science run. That effort will be documented elsewhere.
} 
two main pieces: the $4 \mathrm{~km}$ beam tube arms (through which the laser beams pass between the vertex and end test masses), and the vacuum chambers that house the suspended optics and associated equipment. The vacuum tubing for the arms $[13,14]$ is $1.2 \mathrm{~m}$ in diameter, fabricated from $3 \mathrm{~mm}$ thick 304L stainless steel, processed to reduce the outgassing to very low levels (1 to

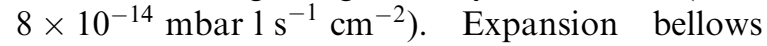
are placed periodically along the arms. An extended bake at elevated temperature was used to remove adsorbed water. The tubing is supported by a ground-level concrete slab, protected by a concrete cover, and aligned to centimeter accuracy [15]. Ion pumps at $2 \mathrm{~km}$ intervals and liquid nitrogen cooled cryogenic traps where the

Table 1

Location and orientation of the LIGO detectors

\begin{tabular}{lll}
\hline LIGO observatory & Hanford & Livingston \\
\hline Vertex latitude & $46^{\circ} 27^{\prime} 18.5^{\prime \prime} \mathrm{N}$ & $30^{\circ} 33^{\prime} 46.4^{\prime \prime} \mathrm{N}$ \\
Vertex longitude & $119^{\circ} 24^{\prime} 27.6^{\prime \prime} \mathrm{W}$ & $90^{\circ} 46^{\prime} 27.3^{\prime \prime} \mathrm{W}$ \\
Orientation of $\mathrm{X}$ arm & $324.0^{\circ}(\mathrm{NW})$ & $252.3^{\circ}(\mathrm{WSW})$ \\
\hline
\end{tabular}

Note that the Livingston Observatory is rotated by $\sim 90^{\circ}$ with respect to the Hanford Observatory, when the earth's curvature is taken into account. arms enter the buildings at the vertex, end, and midstations maintain the base pressure in the arms between $10^{-8}$ and $10^{-9}$ mbar, with the residual gas being mainly hydrogen. This pressure is sufficient to put the residual gas scintillation well below the LIGO design sensitivity.

The seismic isolation system, test masses, and other interferometer optics are housed in vacuum chambers at the vertex, mid-stations (at Hanford), and end-stations. Large gate valves where the beam tubes enter the buildings allow the vacuum chambers to be isolated from the beam tubes and brought to atmospheric pressure for work on the suspended optics while maintaining the vacuum in the $4 \mathrm{~km}$ arms. The vacuum chambers have large doors to aid in the access to install and align optics. When the chambers are at atmospheric pressure they are purged continuously with clean (Class 10) dry air. They have numerous viewports (for laser beams to enter and exit the vacuum system and for video camera monitoring of the interior components) and electrical feedthroughs. The pumping system includes roughing pumps with Roots blowers, and hydrocarbon-free turbopumps. Only ion pumps and cryogenic traps are used when the interferometers are operating. The vacuum chambers are fully instrumented with

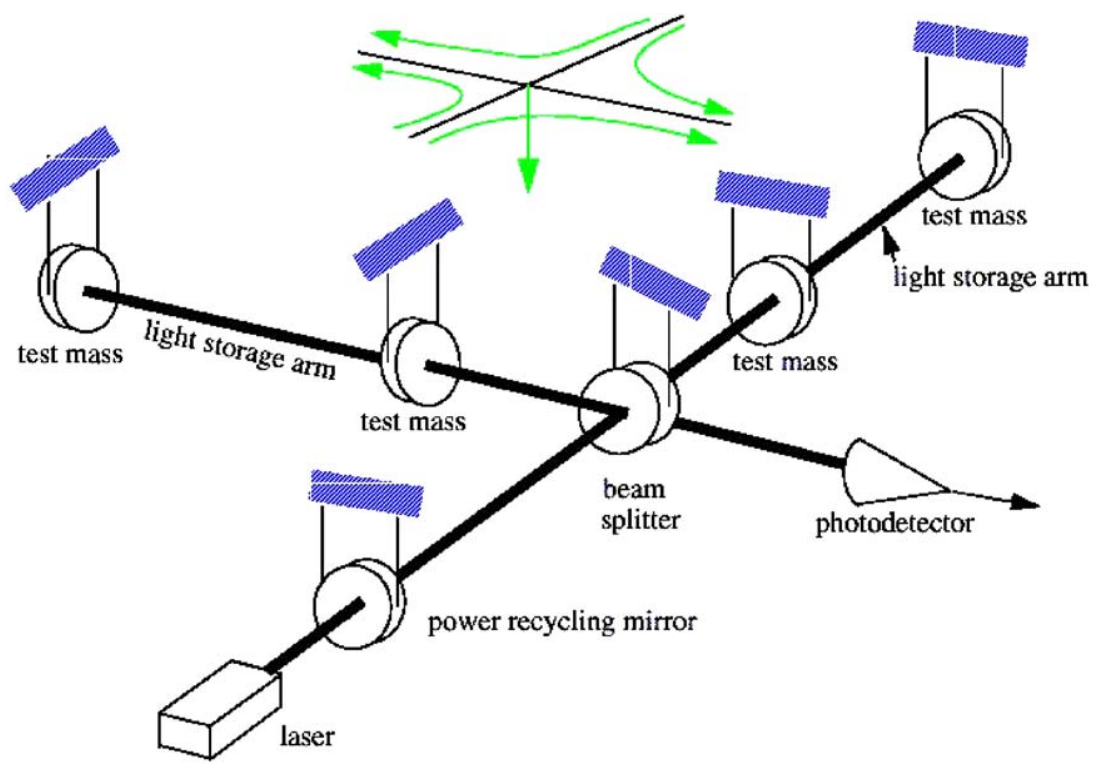

Fig. 1. Schematic layout of a LIGO interferometer. 
gauges and residual gas analyzers; pressures range between $4 \times 10^{-8}$ and $3 \times 10^{-9}$ mbar. All materials used in the vacuum chambers and for the installed detector equipment are carefully processed and screened to minimize the amount of hydrocarbons introduced into the vacuum system as a precaution against mirror contamination [16].

The basic optical configuration of each LIGO detector is that of a power-recycled Michelson interferometer with resonant arm cavities, shown in Fig. 1. Gravitational waves produce strains in space. The arm cavity mirrors serve as the inertial test bodies (test masses), which move in response to these strains. For example a sinusoidal wave incident on the plane of the interferometer will cause an apparent shortening of the optical path along one arm and a lengthening along the other arm, and this process reverses half a cycle later in the signal evolution. Laser light is incident from the bottom-left on the beamsplitter, which divides it and sends it to low-loss cavities in the arms. The transmission of the input mirror in each cavity is much larger than the losses in the cavity, and thus when the cavities are on resonance, almost all of the light is returned to the beamsplitter. The beamsplitter is held so that the light emerging from the antisymmetric port of the interferometer (right) is at a minimum, and almost all of the light is reflected back toward the laser. The power-recycling mirror forms a resonant optical cavity with the interferometer, causing a build-up of power in the recycling cavity. The arm cavity mirrors serve as the inertial test bodies (test masses), moving in response to the gravitational wave.

\subsection{Laser}

Each interferometer is illuminated with a continuous-wave Nd:YAG laser operating in the $\mathrm{TEM}_{00}$ Gaussian spatial mode at $1064 \mathrm{~nm}$, and capable of $10 \mathrm{~W}$ output power [17]. A small portion of the beam is used to stabilize the laser frequency using a reference cavity in an auxiliary vacuum chamber (Fig. 2). The beam going to the reference cavity is double-passed through an acousto-optic modulator driven by a voltage controlled oscillator; this allows an offset between the laser frequency and the reference cavity frequency to permit the laser to follow the arm cavity length change due to tidal strains. This initial level of stabilization is at $0.1 \mathrm{~Hz} / \mathrm{Hz}^{1 / 2}$ or better in the gravitational wave band. The main portion of the beam is passed through a $45 \mathrm{~cm}$ path length triangular cavity to strip off non- $\mathrm{TEM}_{00}$ light and to provide passive filtering of the laser intensity noise with a pole frequency of $1.5 \mathrm{MHz}(0.5 \mathrm{MHz}$ at Livingston for the S1 run). An intensity noise control system designed to reduce relative intensity fluctuations below $10^{-8} \mathrm{~Hz}^{-1 / 2}$ was only partially implemented

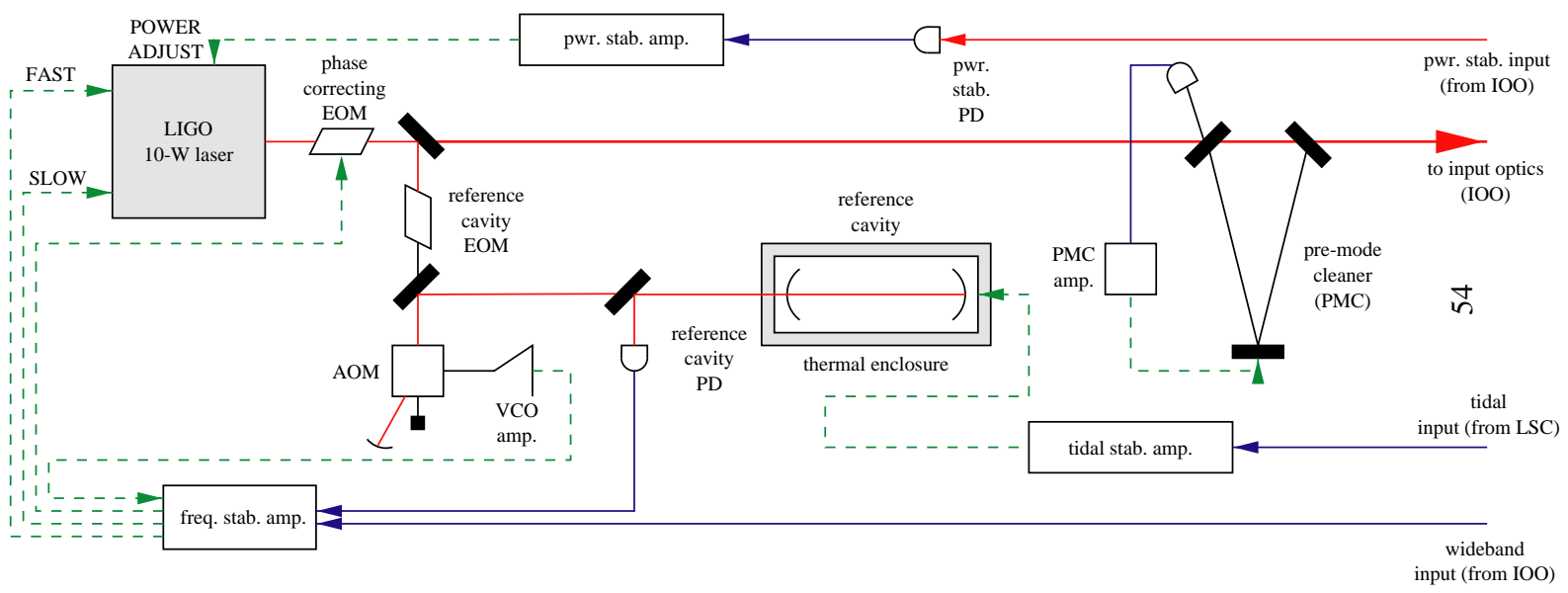

Fig. 2. Simplified schematic of laser stabilization. EOM: Electro-Optic Modulator; AOM: Acousto-Optic Modulator; VCO: Voltage Controlled Oscillator; PD: Photo Diode; PMC: Pre-Mode Cleaner; IOO: Input Optics; LSC: Length Sensing/Control system. 
during the S1 run, leaving the intensity noise at approximately $10^{-7} \mathrm{~Hz}^{-1 / 2}$ level. Electro-optic modulators impress radio-frequency sidebands on the light at 24.5 and $33 \mathrm{MHz}(29.5$ and $26.7 \mathrm{MHz}$ for $\mathrm{H} 2$ ) for sensing respectively the interferometer, and suspended mode cleaner [18] degrees of freedom. The design for the LIGO interferometers is for $8 \mathrm{~W}$ to be incident on the mode cleaner. However, the commissioning of the instrument for high input power was not completed at the time of $\mathrm{S} 1$, and the powers incident on the mode cleaner had been adjusted (through the use of attenuators and reduced laser power) to approximately $1 \mathrm{~W}$ for $\mathrm{H} 1$ and $\mathrm{L} 1$ and approximately $6 \mathrm{~W}$ for $\mathrm{H} 2$.

\subsection{Input optics}

After the laser beam enters the vacuum system, it passes through a set of input optics to condition it before it passes to the main interferometer. First, it passes through a mode cleaner - $\mathrm{a} \sim 24 \mathrm{~m}$ path length triangular ring cavity with a finesse of $\sim 1350$, formed from separately suspended mirrors. This cavity stabilizes the beam size, position and pointing. It also blocks the 33 (or 26.7) $\mathrm{MHz}$ sidebands, but transmits the 24.5 (or 29.5) $\mathrm{MHz}$ sidebands used for the interferometer sensing, which are at multiples of the mode cleaner free spectral range. In addition, it serves as an auxiliary reference for the laser frequency control servo, reducing frequency noise in the transmitted laser light to the $10^{-3} \mathrm{~Hz} / \mathrm{Hz}^{1 / 2}$ level for the $\mathrm{S} 1$ run parameters. After the mode cleaner, the beam passes through a Faraday isolator, which diverts light returning from the interferometer onto a photodetector. This prevents the returning light from reaching the laser and causing excess noise, and allows the common-mode motions of the testmass mirrors to be sensed. Finally, the beam passes through an off-axis telescope formed by three suspended mirrors, which expands the beam to match the $\sim 4 \mathrm{~cm}\left(1 / e^{2}\right.$ radius in power $)$ mode of the arm cavities.

\subsection{Interferometer optics}

The main interferometer optics $[19,20]$ are fabricated from high-purity fused silica, $25 \mathrm{~cm}$ in diameter and $10 \mathrm{~cm}$ thick (except the beamsplitter which is $4 \mathrm{~cm}$ thick). Radii of curvature of the cavity optics are chosen so that the arm cavities have a stability $g=\left(1-L / R_{1}\right)\left(1-L / R_{2}\right)(L$ is the cavity length and $R_{n}$ are the radii of curvature of the two cavity mirrors) of 0.33 ( $\mathrm{H} 1$ and $\mathrm{L} 1$ ) or 0.67 (H2), to minimize the excitation of higher order transverse modes by separating them in frequency from the laser frequency and its RF modulation sidebands. The surface figure accuracy of the polished optic is better than $1 \mathrm{~nm}$; the coatings have a thickness uniformity that holds their contribution to the apparent surface flatness negligible. The coatings have a power absorption less than $1 \mathrm{ppm}$ and scatter less than $70 \mathrm{ppm}$. All optics are wedged (typically about $2^{\circ}$ ) to reduce the possibility of stray reflections interfering with the main beam and to give access to samples of the light inside the interferometer. Transmission of the input mirrors to the arm cavities is $2.7 \%$ and the end mirrors have a transmission of approximately $12 \mathrm{ppm}$, to give an arm cavity pole frequency of $85 \mathrm{~Hz}(170 \mathrm{~Hz}$ for $\mathrm{H} 2)$. The beamsplitter reflectivity was specified as $50 \pm 0.5 \%$. The recycling mirror transmission is also $2.7 \%$, to give a design recycling factor (or increase in the circulating power) of $\sim 50$ for the optics as designed and at full power.

During the S1 run, the low light input power led to the optical configurations of the three interferometers operating away from their design point. At full operating power, absorption of light in the substrate and coating of the input mirrors for the arm cavities is expected to create significant thermal lensing. As a result, the curvature of the recycling mirrors was figured to compensate for this anticipated thermal lensing. Since the incident laser power in the $\mathrm{H} 1$ and $\mathrm{L} 1$ interferometers was significantly under the design level, the lack of thermal lensing makes the recycling cavities slightly unstable for the modulation sideband light. This has little effect on the carrier recycling gain (since the carrier spatial mode is stabilized by resonance in the arm cavities) but reduces the transmission of sideband light to the antisymmetric port by more than a factor of 10 . This further reduces the main differential arm length sensitivity in the high frequency region where shot 
noise is expected to be dominant. In the case of the $2 \mathrm{~km}$ interferometer $\mathrm{H} 2$, although it was receiving nearly the design input laser power, an out-ofspecification anti-reflection coating on the input mirror of one arm caused excess loss in the recycling cavity and reduced the recycling gain for the carrier by more than a factor of two. As a result it also did not develop the required thermal lens and its transmission of sidebands to the dark port was also degraded by a similar factor. These limitations contributed to the relatively high noise level of the instruments seen in the sensing-noise limited regime $(f>200 \mathrm{~Hz})$.

\subsection{Suspensions}

Each interferometer optic is suspended as a pendulum from vibration-isolated platforms to attenuate external disturbances in the gravitational wave band; see Fig. 3 for a schematic drawing. The suspension fiber is a steel piano wire, loaded at approximately $40 \%$ of its yield stress, passing under the optic as a simple loop [48,49]. Small, notched glass rods are glued to the side of the optic a few millimeters above the center of mass to define the suspension point and minimize frictional

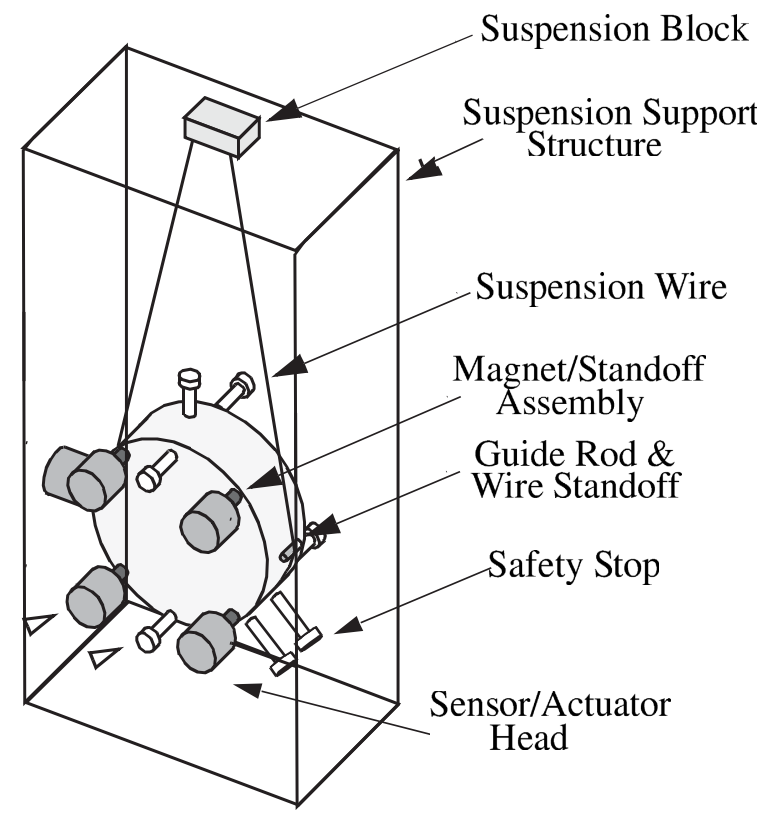

Fig. 3. LIGO suspension. losses. The normal modes of the test mass optic suspension are approximately $0.74 \mathrm{~Hz}$ (pendulum mode), $0.5 \mathrm{~Hz}$ (yaw mode), $0.6 \mathrm{~Hz}$ (pitch mode), $12 \mathrm{~Hz}$ (bounce mode), $18 \mathrm{~Hz}$ (roll mode) and multiples of $345 \mathrm{~Hz}$ (violin mode). Thermal noise is managed in interferometric gravitational wave detectors by placing resonances above or below the detection band when possible, and by choosing materials and assembly techniques which yield high resonance $Q$ 's [21]. This gathers the thermal noise power into a narrow band and lowers the values on either side of the resonance. In the case of the suspensions, high resonance $Q$ 's (measured to be typically 2 to $4 \times 10^{5}$ ) in all suspension modes yield a negligible level of off-resonance thermal noise for the S1 sensitivity.

The suspension system also provides the means for applying control forces and torques to align the mirrors and hold the interferometer in resonance. Four small Nd:Fe:B magnets are attached to the back of the mirror using aluminum stand-offs and a vacuum compatible epoxy, with alternating polarities to reduce coupling to environmental magnetic fields. The suspension structure supports voice coils on ceramic forms near the magnets to produce control forces. Each of these assemblies also incorporates an LED/photodiode pair arranged so that the magnet partially obstructs the path between them (a "shadow sensor"). This provides a read-out of the longitudinal position of the magnet with a noise level of approximately $10^{-10} \mathrm{~m} / \mathrm{Hz}^{1 / 2}$ in the gravitational wave band. Similar magnets are attached to the sides of the optic and a shadow sensor/voice coil assembly acts on one of these to damp sideways motion.

The magnet and coil actuators are driven by several sensors, via servo controllers, allowing control of their positions and orientations with respect to both the local structures and the globally-measured lengths and angles. Local damping of the modes of the suspension is provided by feeding appropriately filtered and mixed signals from the shadow sensors to the coils to create a damping force near the pendulum frequencies. Signals from interferometric-based wavefront sensors and optical levers (described below) are also applied to maintain the pointing of the test mass. Lastly, the interferometer length 
signals are applied to acquire resonance and hold the operating lengths for the interferometer to within $\sim 10^{-13} \mathrm{~m}$ rms. The suspension controllers, which combine and filter these signals appropriately, were of two styles during S1: an original analog system with some digital gain and filter controls (for $\mathrm{H} 2$ and L1), and a system with the signal processing performed digitally [22] (H1). In all cases, a significant low-frequency noise contributor was the final amplifier, which for S1 had to deliver stronger control forces than those expected for the final configuration. This then compromised the gravitational wave-band performance.

Thermal noise internal to the mirrors is minimized by maintaining high $Q$ 's in all the internal modes. The fused silica internal losses are anticipated to make the dominant contribution to thermal noise. However, the dielectric coating on the mirror will also contribute noticeably because of its proximity to the beam [23]. The attachments to the mirror for the suspension and the magnets can degrade the individual modal $Q$ 's but, because of their distance from the front surface of the optic, their effect on thermal noise is negligible. Insitu measurements of $Q$ 's typically range from $2 \times$ $10^{5}$ to $1.6 \times 10^{7}$, depending on the mode. Calculations indicate that the thermal noise is near the design goal and thus negligible for the S1-run sensitivity.

\subsection{Seismic isolation}

The vibration isolation systems are four layer passive isolation stacks [24]. The final stage in each vacuum chamber is an aluminum optical table that holds the optic suspensions. Each optical table is supported by four legs. Each leg consists of a series of three heavy stainless steel cylinders, supported by coil springs made with phosphor bronze tubing containing inner constrained layers which are sealed from the vacuum via electron-beam welding. The transfer function of ground motion to table motion shows a series of broad peaks between 1.5 and $12 \mathrm{~Hz}$, representing the normal modes (typical $Q \sim 10-30$ ) of the masses moving on the springs, followed by a steep falloff above the highest resonance. The total attenuation reaches a value of $10^{6}$ by about $50 \mathrm{~Hz}$. The high $Q$ 's of the resonances in the $1.5-12 \mathrm{~Hz}$ band presents a particular problem at LLO, where they amplify anthropogenic ground noise in this frequency range, and cause difficulties in locking during daylight hours. A planned six degree-offreedom external active isolation system to cope with this excitation was not in place during S1. The support points for the seismic isolation stack penetrate the vacuum chamber through bellows that decouple the seismic isolation stack from vacuum chamber vibrations and drift. External coarse actuators at the support points permit translations and rotations to minimize the control forces that are needed to align the optics during the initial installation and to compensate for any long-term settling.

In addition, the systems at the ends of the arms are equipped with a fine actuator aligned with the arm that can translate the entire assembly (seismic isolation stack and optic suspension) by approximately $\pm 90 \mu \mathrm{m}$ over the frequency range from DC to $\sim 10 \mathrm{~Hz}$. This system is used during the interferometer operation to compensate for earth tides, using a simple predictive model and a very slow feedback from the differential and common mode arm length controls. At LLO, an additional microseismic feed-forward system [25] was used to reduce the length fluctuations of the arms at the microseismic frequency (approximately $0.16 \mathrm{~Hz}$ ). Also, the L1 detector's fine actuators were used together with seismometers in a beam-direction active seismic isolation system at each test mass chamber, which reduced seismic excitation of the most troublesome stack modes by a factor of $\sim 5$.

\subsection{Length and angle control}

There are four longitudinal degrees of freedom that must be held to allow the interferometer to function: the two arm lengths are held at the Fabry-Perot cavity resonance condition, the beamsplitter position is set to maintain the light intensity minimum at the antisymmetric port and the recycling mirror position is positioned to meet the resonance condition in the recycling cavity. These lengths are sensed using RF phase modulation sidebands on the incident light in an extension 


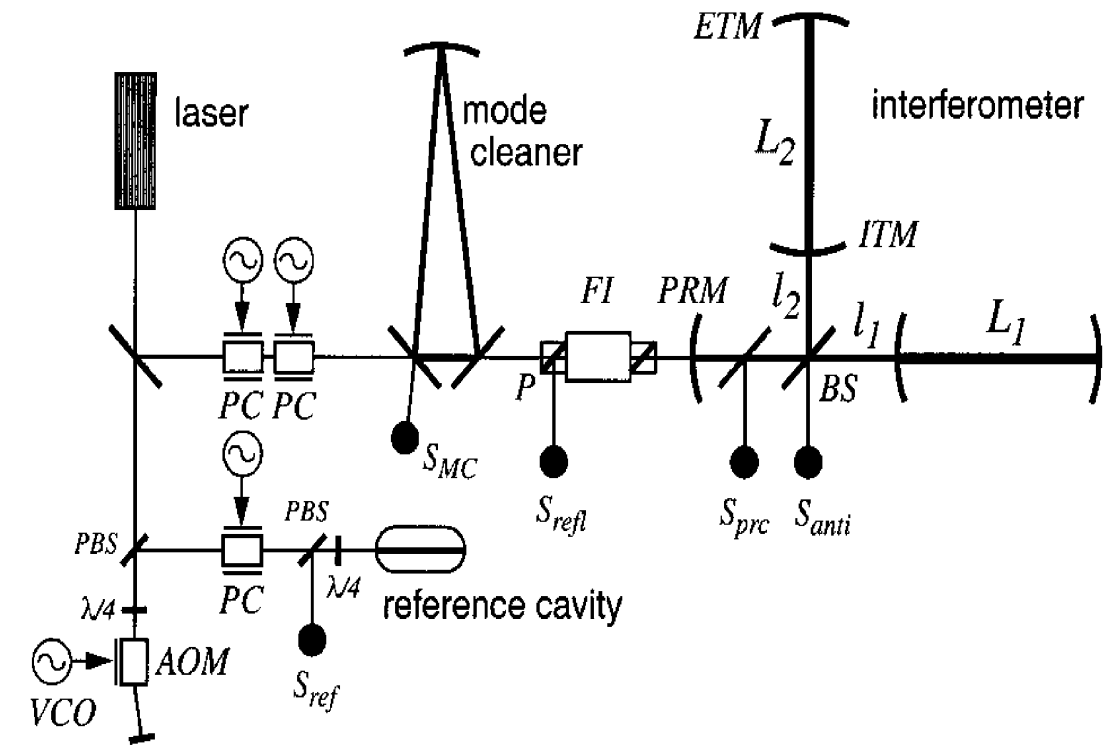

Fig. 4. Schematic drawing of a LIGO interferometer showing laser, input light mode cleaner, and the locations of the photodiodes $\left(\mathrm{S}_{\mathrm{xxx}}\right)$ used to sense and control the resonance conditions. $L_{1}$ and $L_{2}$ are the arm cavity lengths; a gravitational wave produces a differential signal of the form $\left(L_{1}-L_{2}\right)$, and $\left(L_{1}+L_{2}\right)$ is a sensitive measure of the laser frequency noise. The Michelson degrees of freedom are differential $\left(l_{1}-l_{2}\right)$ and common-mode $\left(l_{1}+l_{2}\right)$, the latter measured with respect to the recycling mirror. PBS: Polarizing Beam Splitter. AOM: Acousto-Optic Modulator. PC: Pockels Cell. VCO: Voltage Controlled Oscillator. $\mathrm{S}_{\mathrm{mc}}$ : Signal, Mode Cleaner. $\mathrm{S}_{\mathrm{ref}}$ : Signal, Reference Cavity. $\mathrm{S}_{\mathrm{refl}}$ : Signal, reflected light. FI: Faraday Isolator. PRM: Power Recycling Mirror. S $\mathrm{S}_{\mathrm{prc}}$ : Signal, Power Recycling Cavity. $\mathrm{S}_{\text {anti }}$ : Signal, Antisymmetric Port. BS: Beamsplitter. ITM: Input Test Mass. ETM: End Test Mass.

[26] of the Pound-Drever-Hall technique. The modulation frequency was chosen so that the phase modulation sidebands are nearly antiresonant in the arm cavities; the carrier light is strongly overcoupled so that 0.97 of the light is reflected on resonance, and it receives a $\pi$ phase shift on reflection. By making the recycling round trip cavity length an odd number of RF half-wavelengths, the recycling cavity can be simultaneously resonant for the carrier and sidebands. A small length asymmetry $(\sim 30 \mathrm{~cm})$ is introduced between the beamsplitter and the two input test masses to couple the sideband light out the dark port.

Three interferometer output beams (Fig. 4) are used to determine the longitudinal degrees of freedom [27], which are best thought of as two differential motions (arm cavities or strain readout, and the Michelson), and two common-mode motions (common mode 'breathing' of the arm cavities, and of the power recycling cavity). A photodiode signal at the antisymmetric port is demodulated with the $90^{\circ}$ quadrature of the modulation drive to give a signal proportional to the difference in arm cavity lengths (differential arm length). A second photodiode monitors the light reflected from the recycling mirror (separated from the incident beam by a Faraday isolator); it is demodulated in phase with the modulation drive and is primarily sensitive to the average of length of the two arm cavities (common mode arm length). The third photodiode monitors light from inside the recycling cavity, picked off from the back (anti-reflection-coated) side of the beamsplitter with the aid of the small wedge angle in the substrate. The in-phase signal is primarily sensitive to the recycling cavity length, while the quadrature phase is sensitive to the Michelson path difference from the beamsplitter to the input test masses. One major deviation from the final interferometer design during S1 was that attenuators were placed in front of the antisymmetric port photodiodes on all three interferometers, reducing the effective power used in each interferometer to about $50 \mathrm{~mW}$ instead of the $6 \mathrm{~W}$ nominal value. 
These attenuators protected the photodiodes from saturation, and possible damage, during the commissioning phase before the complete mirror angular controls were implemented and when large fluctuations in the power on the photodiodes were present. This had a particularly significant impact on the performance in the high frequency region (above a few hundred $\mathrm{Hz}$ ), where the low effective light level combined with the reduced sideband efficiency noted above to cause the noise to be well above the design level.

The signals from these three photodiodes, appropriately demodulated and filtered, are used to control the lengths and hold the interferometer in resonance. The high frequency portion of the reflected photodiode signal $S_{\text {refl }}$ is fed back (via an analog path at Hanford for S1, digital at Livingston) to the mode cleaner and laser to stabilize the input laser frequency to the average arm length. The signals $S_{\text {prc }}$ and $S_{\text {anti }}$ from the other two photodiodes are used to control the positions of the interferometer optics. The demodulated signals from all three photodiodes are whitened with an analog filter, digitized with a 16 bit ADC operating at 16384 samples per second, and digitally filtered with the inverse of the analog whitening filter to return them to their full dynamic range. A dedicated real-time signal processor combines these error signals via a matrix (whose coefficients are adjusted in real time during the lock acquisition process) to form appropriate control signals, filters them, and sends the results to combinations of optics to control the interferometer. It also passes the photodiode signals (error signals) and the feedback signals to the data acquisition system. The flexibility of the digital control system to respond in changes to the interferometer response function during the 'locking' process as a function of sensed light levels, and to allow specialized filters to be implemented on the fly, has been crucial to the ability to acquire lock on the interferometers [28], to aid in the commissioning, and ultimately to suppress noise in the control systems.

As noted above, an ensemble of optical levers and wavefront sensors is designed to sense and control the angular degrees of freedom of the suspended optics in the main part of the inter- ferometer [29,30]. Each large $(25 \mathrm{~cm})$ optic is equipped with an optical lever, consisting of a fiber-coupled diode laser and a (position sensitive) quadrant photodiode, which is intended to hold the optic stable while the interferometer is unlocked. These components are mounted on piers outside the vacuum system and operate through viewports at distances between 1 and $25 \mathrm{~m}$ from the optic; their long-term stability and independence from the interferometric sensing system allows a manual alignment to be maintained continuously.

The full instrument design includes a wavefront sensing control system to optimize the alignment during operation. Quadrant photodiodes are placed at the output ports of the interferometer, in the near field and (via telescopes) in the far field. The photocurrents are demodulated as for the length control system, and sums and differences can be formed to develop a complete set of alignment information which is then used to control the mirror angles, using the suspension actuators. However, at the time of the S1 run, this system was only partially commissioned, and only the mode cleaner and two degrees of freedom of the interferometer, the differential pitch and yaw of the end test masses (cavity end mirrors), were controlled by wavefront sensors. As an interim measure, the incomplete wavefront sensing was complemented by signals from the optical levers during operation. However, the optical lever angular sensing noise is much greater than that for the wavefront sensors. Even after careful control-law shaping, the optical levers remained one of the principal contributors to the lowfrequency noise of the instrument for S1.

Baffles to capture stray light are placed along the $4 \mathrm{~km}$ beam tubes, and at specific places near the optics inside the vacuum chambers, to reduce the possibility of a scattered beam or one from an intentional wedge in the optics from recombining with the main beams. Some of the baffles for the final installation were not in place for the S1 run, but calculations indicate that this should not have been a source of noise at our present level of sensitivity.

A noise model of the instruments summarizes the limits to the performance of the interferometer 


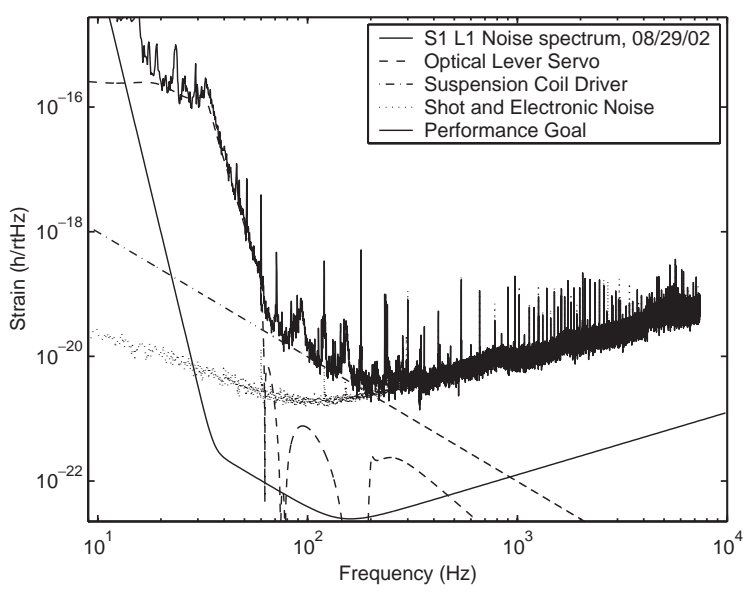

Fig. 5. A frequency-domain model of the noise sources at the time of the S1 run for the Livingston (L1) detector. The noise sources, discussed in the text, are assumed to add in quadrature. The actual noise curve is also shown, along with the performance expected for the instrument when working at the design level.

at the time of this science run. The model for the Livingston detector is shown in Fig. 5. In general, the contributions are evaluated by measuring a source term (e.g., laser frequency noise), and measuring a coupling function (e.g., the transfer function from an intentional frequency modulation to the response in the strain channel), and then multiplying these two together to make a prediction. In some cases, analytical models are used (for example the mechanical $Q$ of the suspension systems is measured and then used in a model of the thermal noise contribution). For this model, all the terms are considered to be independent, and the noises are added as the square root of the sum of the squares. Many sources of noise have been modelled; this figure only shows those that limit the present performance. The model explains the overall instrument noise performance well, and subsequent commissioning efforts have shown that reductions in the leading noise terms also leads to the anticipated reduction in the overall instrument noise.

\subsection{Simulations}

In addition to subsystem dynamics and control models, two simulations played a significant role in the design and commissioning of the LIGO detectors. The first is an FFT-based optical propagation code [31] that models the powerrecycled Michelson interferometer with FabryPerot arms. This code was used to develop the specifications for the interferometer optics, and has been used in comparisons with commissioning data to evaluate the performance of the optics as installed. The second simulation is an end-to-end time-domain simulation of the LIGO interferometers [32]. This model includes a modal-based optical propagation, accurate modeling of the electronic feedback, simplified models for the suspension systems, and typical noise inputs. This model proved to be invaluable in developing the lock acquisition software.

\subsection{Environmental monitoring}

A system of auxiliary sensors is installed at each LIGO site to monitor possible environmental disturbances. The Physics Environment Monitor system (PEM) contains seismometers and tiltmeters to monitor low frequency ground disturbances, accelerometers and microphones to monitor higher frequency mechanical disturbances, magnetometers to monitor magnetic fields that might affect the test masses, and monitors of the line power. Sensors are present in all buildings and near all key sensitive components. They have been used to e.g., help identify sources of acoustic and electromagnetic coupling, and to help design improvements to the apparatus; as the instrument sensitivity improves, they will be used as veto signals in the astrophysical analyses. Planned cosmic ray detectors and $\mathrm{rf}$ monitors were not operational at the time of the S1 run.

\subsection{Control and data systems}

Supervisory control of the interferometers is accomplished using Experimental Physics and Industrial Control System (EPICS) [33]. EPICS establishes a communications protocol within a non-hierarchical computer network and provides an operator interface from networked workstations located in the control room. Processors distributed in all electronics racks can modify 
amplifier gains, offsets, filtering, on/off controls, etc., allowing either manual or automated (scripted) control of the state of the electronics. The EPICS processors also interface to analog-todigital converters to provide monitors for the electronics inputs and outputs. Each interferometer has approximately 5000 EPICS variables (either control or monitor points). EPICS also provides tools for capturing and restoring the state of the instrument to ensure that this complex instrument can be reliably brought to a known configuration.

The data acquisition and control system collects signals from the interferometer and from the environment, and delivers signals for the length and angle controls. VME-based converters and processors are used, and acquisition systems are placed in the vertex building and the mid-and endstations. Analog signals are digitized with 16-bit resolution. Fiber optics are used to link the instrument racks together, and a shared memory approach allows data to be collected and shared by a number of systems over the multi-km distances. The data are collected with 16-bit resolution. The complete data are formatted into the standard data 'Frames' (a format used by all of the interferometric gravitational wave community) and initially are stored on spinning media for a quick 'look-back' buffer of roughly 2 weeks. All data are archived to tapes for later analysis. Reduced data sets also in the standard Frame format, configured for a given science run, are produced as well; these serve most analysis needs.

It is important that the data acquisition system accurately time-stamp the data it records. The fundamental timing for both sites is derived from GPS receivers located at each building (vertex, mid and end). A $2^{22} \mathrm{~Hz}$ (approximately $4.2 \mathrm{MHz}$ ) clock signal is generated from the GPS as well as a 1 pulse-per-second synchronization signal. Together these are used to synchronize the data collected by the various processors. Ramp signals are used to monitor any timing errors and alarms are set for the operators. This monitoring has proven useful during S1. It showed that the timing was subject to jumps (typically 10's of milliseconds, but sometimes larger) when the length control system processors were rebooted, with the consequence that some S1 data had to be eliminated from some analyses because of uncertain timing (These timing jumps have since been cured, and a redundant and independent atomic clock reference is being implemented for the future).

\subsection{Diagnostics and monitoring}

Two closely related systems, the Global Diagnostic System and the Data Monitoring Tool, provide the instrument operators and scientific monitors with tools for evaluating interferometer performance both during commissioning and scientific running [34]. The Global Diagnostic System (or GDS) can access data from any signal collected by the data acquisition system, including test-point signals that can be stored for postanalysis if indicated. It can display the time series and the power spectrum for individual signals, and the transfer function and coherence for pairs of signals. The GDS also has the ability to apply arbitrary-waveform excitations to various test points within the interferometer control systems. These can be used to measure transfer functions through stimulus-response testing. The data can be filtered, decimated, calibrated, stored and recalled for comparisons.

The Data Monitoring Tool (or DMT) is a package of software components running on a set of processors on a dedicated network. A highspeed connection to the data acquisition system makes the full data set available with only a onesecond latency. The emphasis in the DMT is on relatively simple measures of instrument performance applied to the full data stream in realtime. Thus it can give the operators and scientific monitors rapid feedback about interferometer performance. These include such measures as the non-stationarity and burst-like behavior of various types in the interferometer outputs, band-limited rms amplitudes of interferometer outputs and environmental monitors, monitors of calibration lines, histograms to monitor the gaussianity of the data, and real-time estimates of detector sensitivity to neutron star binary inspiral events. The DMT is also an important element of the data analysis process, analyzing the auxiliary channels for veto signals in parallel with the strain channel analysis. 


\subsection{Data analysis system}

To analyze the large volume of data generated, LIGO has developed the LIGO Data Analysis System (LDAS). The LDAS provides a distributed software environment with scalable hardware configurations to provide the computational needs for both on and off site data analysis. The architectural design of the system is based on the concept of multiple concurrent data analysis pipelines in which data is fed into the pipeline as it is collected and proceeds down the pipeline where necessary signal analysis procedures are applied depending on the particular type of analysis that is being carried out [35].

LDAS is complemented by the LIGO/LSC Algorithm Library, which is a set of C-language routines that can run under LDAS or be used independently. They are carefully vetted to ensure that the algorithms and results are correct.

The LDAS distributed software environment is composed of roughly 12 modules called LDAS Application Programming Interfaces (APIs), each of which is a separate process under the Unix operating system. Each module is designed to carry out the multitude of steps associated with each unique pipeline. For example, one module has computational elements for reading and writing LIGO channel data in the Frame format, another module has computational elements for signal processing in the time or frequency domain, and another module has computational elements necessary to perform parallel analysis across a cluster of tightly networked CPUs [36]. Upon completion of the data analysis pipeline, data products and results are stored to disk or inserted into the LIGO relational database. The database has tables designed to capture results associated with detector characterization, on line and off line astrophysical searches and multi-detector analyses. The software can be scaled to run on a wide variety of computing hardware.

During the first LIGO Science Run, the LDAS at the LIGO Observatories and data analysis centers located at Caltech and MIT, LDAS at other institutions, and other configuration-controlled computational systems were operated with commissioning configurations of the hardware and software. The software was in the late stage of beta development, having a complete set of modules. The hardware systems consisted of a complement of servers with tens of terabytes of disk storage for the raw data and the LIGO database, along with scaled down computation centers with approximately 200 megaflops of aggregate computational performance between them. In its final configuration, the LDAS hardware will include upgrades to the current servers, and expanded high performance computation clusters with over two teraflops of aggregate computational performance. In addition, new tape storage systems will be put on line which will provide adequate storage at the observatories for six months of local data and storage for all of the data at Caltech; this is where the data for the multiple detectors are brought together. The software is expected to double in performance as we upgrade from beta versions to the first completed version later this year. In addition, the software is being adapted to support Grid Computing technology and security protocols allowing for LIGO data analysis once the computational Grid is deployed in the near future [37].

\subsection{LIGO data}

The full data stream from each of the LIGO interferometers consists of several thousand channels, recorded at rates from 1 to $16384 \mathrm{~Hz}$ with a total data rate of $5 \mathrm{MB} / \mathrm{s}$ per interferometer. These channels include EPICS process variables that define the state of the interferometer, signals from environmental monitors, signals from auxiliary servos in the interferometer (for example, optical lever signals), as well as the main gravitational wave signal. For the servos not operational during S1, the corresponding data channels were recorded, but of course they contain only zeros. The non-gravitational wave data channels can be used in a number of ways:

(1) They can be used to determine the operational "health" of the interferometer (how well it was aligned, whether large offsets were present in any servos, etc.).

(2) They can be used to regress noise from the main gravitational wave channel (for example, 
measurements of the laser frequency noise can be used to correct the gravitational wave channel to remove any residual effects from laser frequency noise coupling to mismatches in the arms).

(3) They can be used to veto non-gaussian noise in the interferometer (for example seismometer data could be used to keep noise from impulsive seismic disturbances from being misinterpreted as a gravitational wave).

At this point in the commissioning, few of these techniques have been explored and developed. In part, this is because the majority of the noise sources in the interferometer at present are attributable to electronic noise entering through imperfect tuning, and consequently, few of the auxiliary channels are expected to be useful. The DMT capabilities to perform this analysis were exercised in preparation and performed very well.

The main signal for the analysis to search for gravitational wave signals is the output of the photodiode at the antisymmetric port, demodulated in the quadrature phase at $24.5 \mathrm{MHz}(\mathrm{H} 1$ and $\mathrm{L} 1)$, or $29.5 \mathrm{MHz}(\mathrm{H} 2)$. This analog signal is amplified and digitized. An analog filter whitens the signals before digitization, and a precise inverse of this filter 'de-whitens' the signal in the digital domain, to best take advantage of the dynamic range and noise in the Analog-to Digital Converter (ADC). Since it is the error point in the servo control system which holds the differential arm length, its interpretation requires correction for the loop gain of the servo. This signal represents the phase difference of the light from the two arms, filtered only by a roll-off at high frequencies because of the arm cavity storage time, and while the interferometer is operating, is a continuous measure of the differential strain between the two arms and thus potentially of gravitational wave signals.

\section{The GEO detector}

The GEO Detector is situated at the perimeter of an agricultural research station to the south-east of Hannover, Germany; see Table 2. The buildings are intended to be just sufficient to accommodate
Table 2

Location and orientation of the GEO 600 detector

\begin{tabular}{ll}
\hline Vertex latitude & $52^{\circ} 14^{\prime} 42.5^{\prime \prime} \mathrm{N}$ \\
Vertex longitude & $9^{\circ} 48^{\prime} 25.9^{\prime \prime} \mathrm{E}$ \\
Orientation of North arm & $334.1^{\circ}$ (NNW) \\
Orientation of East arm & $68.4^{\circ}$ (ENE) \\
\hline
\end{tabular}

Note that the arms form an angle of $94^{\circ} 19^{\prime} 53^{\prime \prime}$. This deviation from perpendicular has negligible effect on the sensitivity.

the instrument and its acquisition and control hardware. Data recording, and much of the operation and on-line monitoring of the instrument, will be performed at the Max Planck Institute in downtown Hannover, once continuous science operation is underway. A microwave link maintains a high-bandwidth dedicated connection between the two.

One central building ( $13 \mathrm{~m} \times 8 \mathrm{~m}$ in size $)$ and two end buildings $(6 \mathrm{~m} \times 3 \mathrm{~m})$ accommodate the vacuum chambers $(2 \mathrm{~m}$ tall, $1 \mathrm{~m}$ in diameter) in which the optical components are suspended. In the central building, nine vacuum chambers form a cluster which can be subdivided into three sections to allow mirror installation without venting the whole cluster. This arrangement allows a minimum of down-time for a change of the signalrecycling mirror (to change the detector bandwidth). To avoid fluctuations of the optical path caused by a time-varying index of refraction, all light paths in the interferometer are in a highvacuum system. For this purpose GEO 600 uses two $600 \mathrm{~m}$ long vacuum tubes of $60 \mathrm{~cm}$ diameter which are suspended in a trench under ground. A novel convoluted-tube design, allowing a wall thickness of only $0.8 \mathrm{~mm}$, was used to reduce weight and cost of the stainless-steel vacuum tube.

The whole vacuum system, except for the mode cleaner and signal recycling section, is pumped by four magnetically levitated turbo pumps with a pumping speed of $1000 \mathrm{l} / \mathrm{s}$, each backed by a scroll pump $\left(25 \mathrm{~m}^{3} / \mathrm{h}\right)$. Due to the use of stainless steel with a low outgassing rate, a 2 day air bake at $200^{\circ} \mathrm{C}$ and a 5 day vacuum bake at $250^{\circ} \mathrm{C}$, a pressure of $1 \times 10^{-8}$ mbar can be achieved in the tubes. Large gate valves allow the beam tubes to be temporarily closed off and maintained under vacuum whenever the instrument vacuum chambers are opened for installation work. Additional 
dedicated pumping systems are used for the mode cleaner section and for the signal-recycling section. The pressure in the vacuum chambers is in the mid $10^{-8}$ mbar range. Great care was taken to minimize contamination of the all-metal vacuum system by hydrocarbons. For this reason the seismic isolation stacks, which contain silicone elastomer and other materials containing hydrocarbons, are sealed by bellows and pumped separately. Furthermore, the light emitting diodes (LEDs), the photodiodes and the feedback coils used as 'shadow' sensors and actuators in the pendulum collocated damping and actuation systems are encapsulated in glass.

The buildings of GEO 600 are split into three regions with different cleanroom classes: the socalled gallery where people can visit and staff can work with normal clothes, the inner section which has a cleanroom class of 1000 and a movable cleanroom tent installed over open chambers with a cleanroom class 100 .

\subsection{Suspension and seismic isolation}

Two different types of seismic isolation and suspension systems are implemented in GEO 600. The first one, used to isolate the mode cleaner optics, consists of a double pendulum suspended from a pre-isolated top-plate. To avoid an excitation of the pendulum mode, four collocated control systems measure the motion of the intermediate mass with respect to a coil-holder arm which is rigidly attached to the top plate, and feed back to the mirror via a coil-and-magnet system.

The seismic isolation system used to isolate the test masses, beamsplitter, and the other mirrors of the Michelson interferometer consists of a triple pendulum [9] suspended from a pre-isolated platform. Each pendulum chain consists of the optic suspended from an intermediate mass which is in turn suspended from an upper mass. Two cantilever spring stages are included in the pendulum design (in the support of the upper and intermediate masses) to reduce the coupling of seismic motion in the vertical direction to the mirror. As in the case of the mode cleaner pendulums, collocated feedback systems are used to damp all six degrees of freedom of the upper pendulum mass and through cross-coupling the other solid-body modes of the multiple pendulum system. The control forces for the length and alignment control are applied from a reaction pendulum which consists of a similar triple pendulum suspended $3 \mathrm{~mm}$ behind the corresponding mirror. The intermediate mass of the reaction pendulum carries coils which act on magnets glued to the intermediate mass of the mirror triple pendulum. To keep the internal quality factor of the mirrors as high as possible, no magnets are glued to the mirror itself, but electrostatic feedback between the mirror and the lowest mass of the reaction pendulum is used to apply feedback forces in the high frequency control band.

\subsection{Suspension}

To further minimize the mechanical losses and thus internal thermal noise of the mirrors and the pendulums, the lowest pendulum stage consists entirely of fused silica; see Fig. 6. The mechanical quality factor $Q$ of fused silica suspensions smaller than [50-52] and more comparable in size has been demonstrated [38] to be greater than $2 \times 10^{7}$. Small fused-silica pieces are attached to the intermediate mass and to the mirror itself by hydroxide-catalysis bonding [39]. This technique provides high-strength bonds and allows the high quality factor to be maintained and therefore the thermal noise to be kept low. Four fused-silica fibers of $270 \mu \mathrm{m}$ diameter each are welded to these fused-silica pieces and support the mirrors.

The optical layout of GEO 600 (see Fig. 7) can be divided into four major parts: The laser system, the input optics, the dual-recycled Michelson interferometer, and the output optics followed by the main photodetector. Some steering mirrors, electro-optical modulators and Faraday isolators are omitted in Fig. 7. All optical components but the laser system and the photodetector are suspended inside the vacuum system.

\subsection{Laser}

The GEO 600 laser system [40] is based on an injection-locked laser-diode pumped $\mathrm{Nd}$ :YAG 


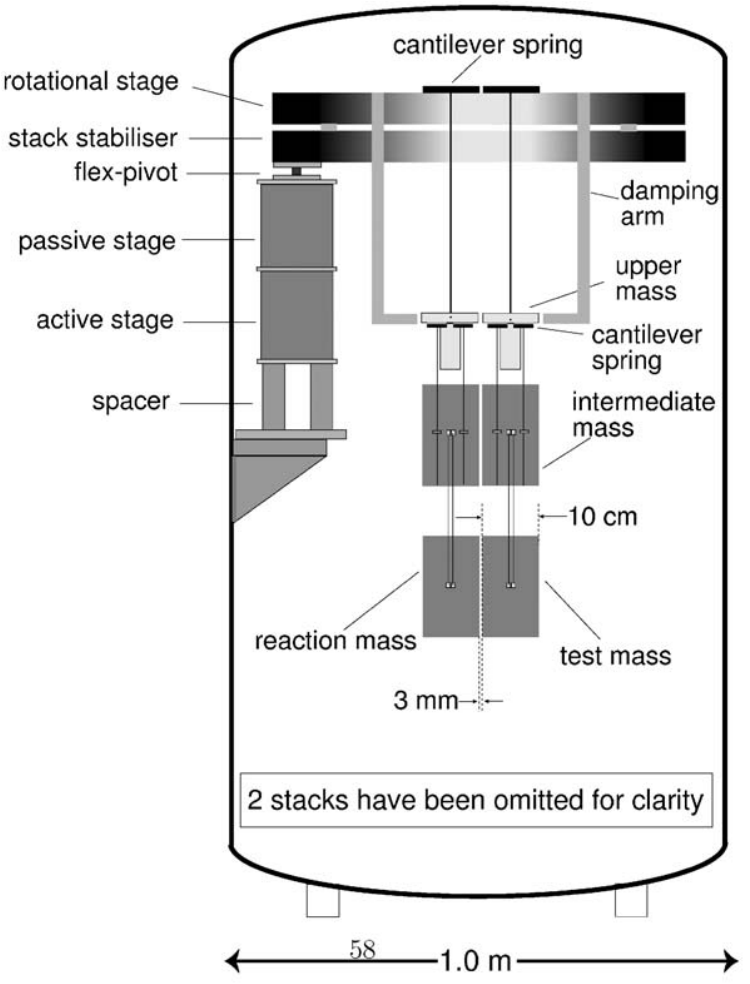

Fig. 6. An outline sketch of the test mass suspensions in GEO 600. The test mass and intermediate mass are made of fusedsilica and are connected by 4 fused-silica fibres. The reaction mass is also of fused-silica and has the electrode pattern required to allow electrostatic actuation forces to be applied to the test mass. The other masses are fabricated from metal. Two stages of vertical isolation are provided in the form of cantilever mounted blade springs. Active local damping is provided from a structure (not shown) held at the upper mass level by the damping arms. The pendulum chains are suspended from a structure which allows crude angular alignment of the mirror, and this is in turn supported by 3 vibration isolating legs. Each leg consists of an active layer and a passive layer which, to avoid contamination of the vacuum are enclosed in steel bellows. A flex-pivot is then required to provide rotational compliance. The reaction chain is omitted from the test mass suspensions in the end stations.

system with an output power of $12 \mathrm{~W}$. A nonplanar ring-oscillator (NPRO) with an output power of $0.8 \mathrm{~W}$ is used as the master laser. The slave laser is formed by a four mirror cavity with two Nd:YAG rods serving as gain media. Each crystal is pumped by fiber-coupled laser diodes with a power of $17 \mathrm{~W}$. Two Brewster plates are incorporated in the slave cavity to define the polarization direction, reduce depolarization losses and compensate for the astigmatism introduced by the curved mirrors of the slave resonator. Due to the mode-selective pumping scheme more than $95 \%$ of the light leaving the laser is in the fundamental $\mathrm{TEM}_{00}$ mode. The fully automated injection locking control servo system acquires lock within $100 \mathrm{~ms}$ and allows stable operation of the laser system.

\subsection{Input optics}

The light from the laser system is passed in transmission sequentially through two triangular resonant cavities, serving as frequency references and optical mode cleaners; they are of 8.0 and $8.1 \mathrm{~m}$ round-trip lengths. The laser frequency is stabilized to the resonant frequency of the first mode cleaner MC1 [10]. For this purpose radio frequency phase modulation sidebands are impressed on the laser beam prior to entering the first mode cleaner. The light reflected by the input mirror of the first mode cleaner interferes with the light leaking out of the first mode cleaner on a quadrant photodiode. The demodulated sum of the photocurrents of all quadrants of this photodiode is used in the Pound-Drever-Hall scheme to develop an error signal for the deviation of the laser frequency from a mode cleaner resonance frequency. This signal is fed back to the master laser frequency actuators and to a phase correcting Pockels cell to stabilize the laser to the first mode cleaner length. With this first control loop in place, the laser frequency will change as the length of $\mathrm{MC1}$ changes. Due to this effect, the lengthcontrol actuator of $\mathrm{MCl}$ can be used to bring the laser/MC1 unit into resonance with the second mode cleaner MC2. For this purpose another pair of $\mathrm{rf}$ sidebands is imposed on the laser beam by an electro-optical modulator, located between the two mode cleaners. The light reflected by the input mirror of the second mode cleaner is aligned onto a quadrant photodetector and the sum of the photocurrents of all segments is demodulated to produce an error signal for this feedback loop. A third control loop is used to bring the laser/MC1/ MC2 unit into resonance with the power-recycling cavity. A Faraday isolator is used between the 


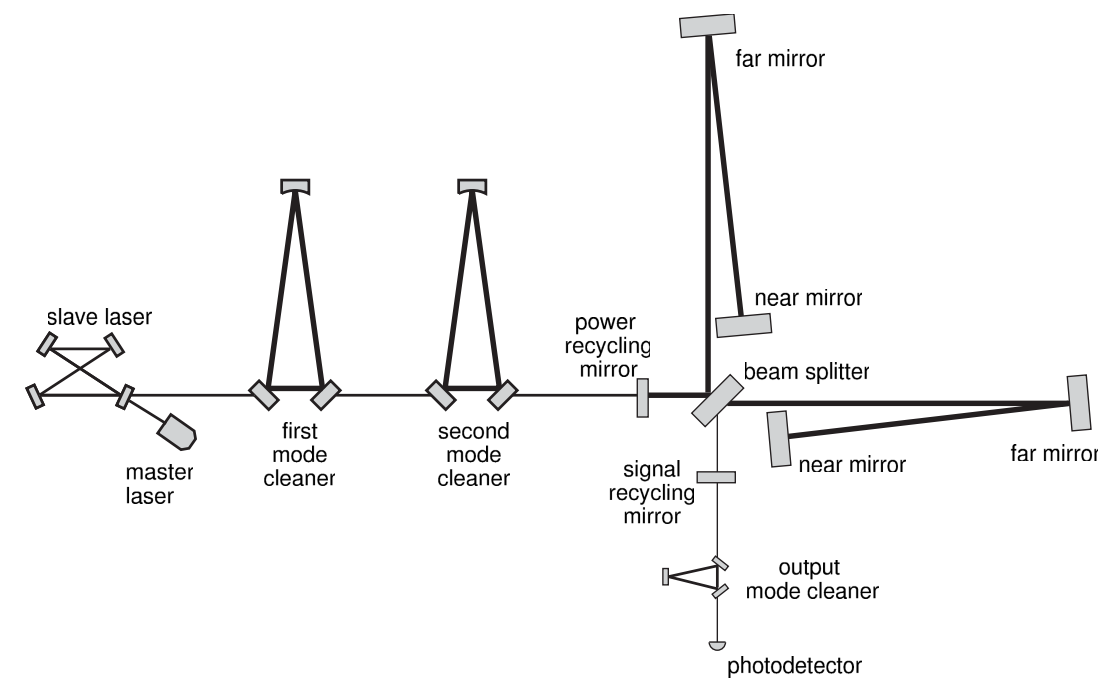

Fig. 7. Optical Layout of GEO 600. A $12 \mathrm{~W}$ injection locked laser system is filtered by two sequential mode cleaners and injected into the dual (power and signal) recycled interferometer. Only power recycling was used for the S1 run. A folded light path is used to increase the round-trip length of the interferometer arms to $2400 \mathrm{~m}$. An output mode cleaner will be used to spatially clean the laser mode before it reaches the photodetector.

mode cleaner and the power recycling mirror to obtain access to the light reflected by the power recycling cavity which is detected on a quadrat photodiode. A detailed description of the frequency control scheme of GEO 600 is given in Ref. [12].

All the quadrant photodiodes mentioned above are used both for length control and for wavefront sensing (and thus alignment control) of the mode cleaners and the power recycling cavity. The difference of the photocurrents of a combination of the quadrants of these diodes is demodulated at the respective $r f$ frequency and the resulting signals provide alignment information of the incoming beam relative to the eigenmode of the relevant cavity. A telescope is used to get near field and far field information of the phase-front differences which can be converted into tilt or rotation as well as $x$ or $y$ parallel shift information of the incoming beam relative to the cavity axis $z$. The appropriate linear combination of these signals is fed back to the mode cleaner mirrors. The alignment error signal of the power recycling cavity is used to change the tilt/rotation of the power recycling mirror and of a steering mirror to keep this cavity aligned to the incoming beam. The complete automatic alignment system [11] uses additional quadrant diodes behind several mirrors to keep the spot positions centered on all the relevant cavity mirrors.

\subsection{Interferometer configuration}

The main interferometer is designed as a dualrecycled folded-arm Michelson interferometer [7,8]. Power recycling leads to a power buildup in the interferometer and improves the shot-noise limited sensitivity of the detector. The anticipated power buildup in GEO 600 is 2000 which results in a power of about $10 \mathrm{~kW}$ at the beamsplitter. Any differential phase change of the light in the interferometer arms (the signature of a gravitational wave) will lead to a change in the light intensity at the output port of the interferometer. The partially transmitting signal recycling mirror will reflect most of this light back into the interferometer and forms another Fabry-Perot cavity, the signal-recycling cavity. In this cavity, the light power representing the signal is enhanced through resonance in a frequency range determined by the cavity bandwidth of the signal recycling mirror and the resonant frequency of the signal-recycling cavity. 
This effect reduces the shot-noise-equivalent apparent displacement noise of the detector for these frequencies. For the S1 run, the signal recycling mirror was not yet installed, and so the instrument ran as a power-recycled folded-arm Michelson.

In the final optical configuration GEO will use an output mode cleaner as a spatial filter of the main interferometer output beam, placed just before the antisymmetric photodetector. The output mode cleaner will be installed when the signal recycling mirror is incorporated, but was not needed for the S1 run.

The length and alignment control systems for the Michelson interferometer and the signal recycling cavity use similar techniques as described above for the mode cleaners and power recycling cavity. Quadrant photodiodes sense the beam at the interferometer output port for the Michelson control. A small fraction of the light in one interferometer arm is reflected by the anti-reflection coating of the beamsplitter and is used for the control of the signal recycling degrees of freedom. The two pairs of sidebands needed for the sensing scheme are impressed on the laser beam injected into the power recycling cavity, and the rf frequencies were chosen to be multiples of the free spectral range of the power recycling cavity. Magnet and coil actuators at the intermediate mass of the suspensions, and electro-static actuators at the mirror level of the triple pendulum suspensions, are used as actuators for the length and alignment control loops.

GEO 600 has five suspended cavities and the suspended Michelson interferometer which need length and alignment control systems. Thirty pendulums need local damping of at least 4 degrees of freedom and additional feedbackcontrol systems are needed for the laser stabilization. Most of these control loops are implemented with analog electronic controllers with some guidance by a LabVIEW computer-control environment [41]. Only the active seismic isolation and some slow alignment-drift-control systems are implemented as digital control loops. The LabVIEW computer system controls pre-alignment, guides lock acquisition of the laser and the mode cleaners, monitors the detector status, and compensates for long-term drifts. Typical response times of this system are $100 \mathrm{~ms}$. The lock acquisition of the recycling cavities and the interferometer is guided by a micro-controller to allow for faster response times.

Although only the light at the detector antisymmetric output includes a possible gravitationalwave signal with a high signal to noise ratio, a multi-channel data acquisition system is needed to detect environmental and detector disturbances and exclude false detections. Two different sampling rates $(16384$ and $512 \mathrm{~Hz})$ are used in the data-acquisition system (DAQ) of GEO 600. In the central building 32 fast channels and 64 slow channels are available, and in each of the end buildings 16 fast channels can be recorded. Most of these channels will be used for detector characterization only. The data are recorded into the standard Frame format for later analysis.

\section{The S1 run}

The S1 run took place from 23 August 2002 15:00 UTC through 9 September 2002 15:00 UTC. The total duration of 17 days spanned three weekends and one national holiday in the U.S., which helped reduce the time lost due to anthropogenic noise sources, particularly at the LIGO Livingston site. Locked times and duty cycles for the four individual interferometers are given in Table 3, along with the double and triple coincidence times for the LIGO detectors. The duty cycle of GEO $600(98 \%)$ is so high that its coincidence time with any combination of LIGO

Table 3

Locked times and duty cycles for the S1 Run

\begin{tabular}{lll}
\hline $\begin{array}{l}\text { Detector/ } \\
\text { combination }\end{array}$ & $\begin{array}{l}\text { Detector hours } \\
\text { coincidence }\end{array}$ & $\begin{array}{l}\text { Locked/duty cycle } \\
(\%)\end{array}$ \\
\hline LIGO H1 & 235 & 57.6 \\
LIGO H2 & 298 & 73.1 \\
LIGO L1 & 170 & 41.7 \\
GEO 600 & 400 & 98 \\
H1-H2 & 188 & 46.1 \\
H1-L1 & 116 & 28.4 \\
H2-L1 & 131 & 32.1 \\
H1-H2-L1 & 96 & 23.4 \\
\hline
\end{tabular}


interferometers is essentially the same as that of the LIGO interferometer(s) alone.

\section{1. $L I G O$}

Each LIGO interferometer had a defined operating state, including which servos were operational, their gains, acceptable light levels on photodiodes, etc. The instrument operator on duty was responsible to lock the interferometer and put it into the required configuration, assisted by computer scripts that set the majority of the parameters. When the desired state was achieved, the operator issued a command that put the interferometer into "Science mode", effectively declaring that the detector was in the proper configuration. At that time, personnel were restricted from entering the experimental halls. A computer program began monitoring the computer control network for any unauthorized changes to the interferometer state, and if any were detected, it automatically removed the interferometer from Science Mode and raised an alarm.

Only data taken in Science Mode segments longer than $300 \mathrm{~s}$ were deemed suitable for analysis. However, because of the still incomplete state of the commissioning, the designation of an interferometer as being in Science Mode was not sufficient to ensure that the data were of uniform quality. Thus each data analysis effort independently evaluated the Science Mode data (assessing, for example, the noise level or the quality of the calibration) and made their own selection of data for further analysis, based on the particular requirements of that analysis.

The LIGO noise level for S1 shown in Fig. 8 is substantially above the design goal. At high frequencies, most of the extra noise can be attributed to the fact that the interferometers were effectively using very low laser power, and using the detection sidebands inefficiently, as described above. This leads to a poorer sensing resolution

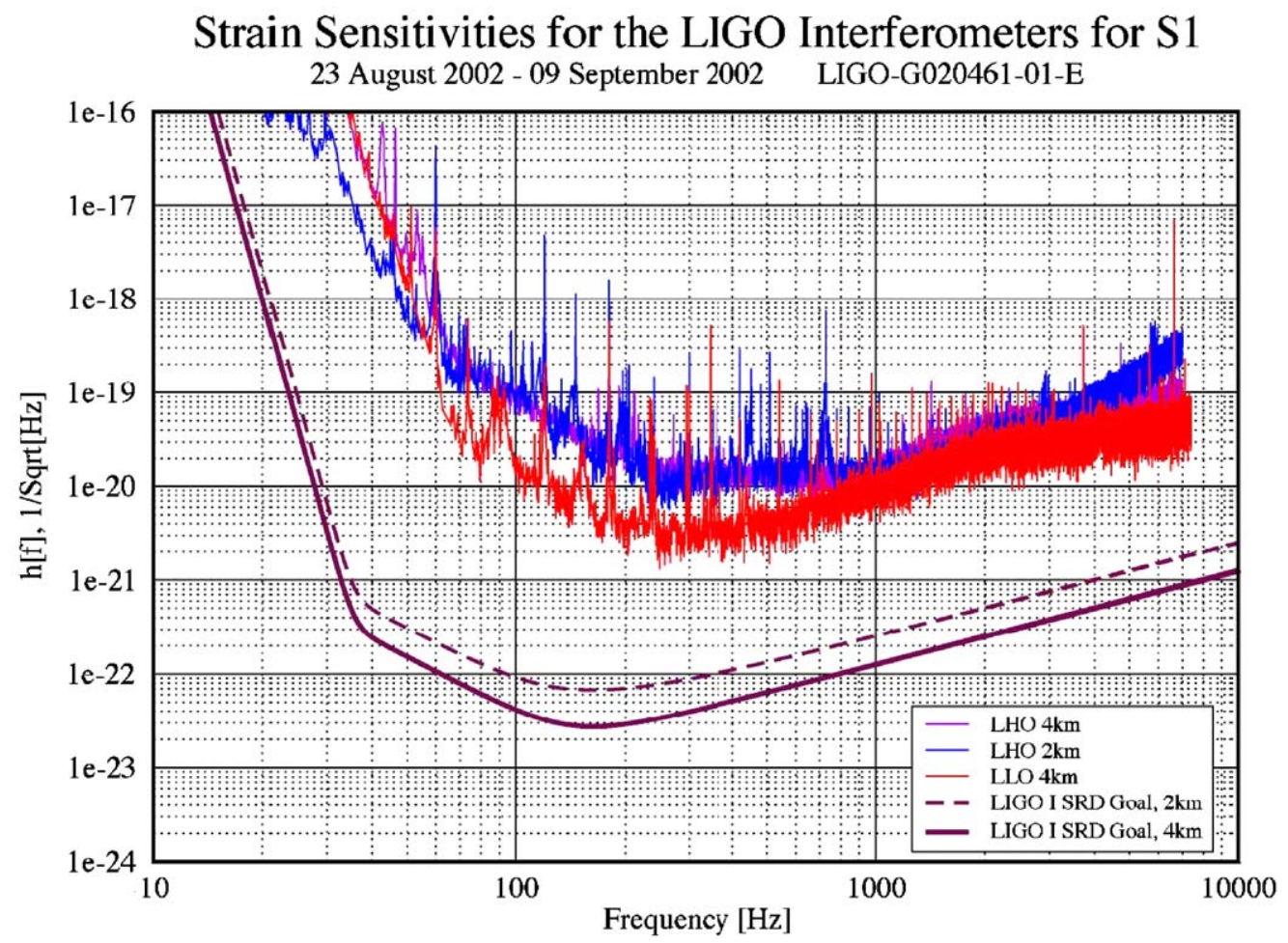

Fig. 8. The LIGO interferometer sensitivities for S1. The LHO $4 \mathrm{~km}$ instrument is $\mathrm{H} 1$; the LHO $2 \mathrm{~km}$ is H2; and LLO $4 \mathrm{~km}$ is L1. The 'SRD Goal' refers to the design sensitivity for the LIGO instruments. 
due to shot noise and electronics noise. At low frequencies, the excess noise is mainly due to noise in the control systems, typically from auxiliary control systems such as angular controls. Much of this noise is due to the incomplete commissioning of the alignment system, and control system filters which are not yet optimized.

The numerous peaks in the spectrum are due to a number of different sources. Multiples of $60 \mathrm{~Hz}$ are prominent (at the $10^{-19}$ level in strain), in part due to switching power supplies that are scheduled for replacement. Acoustic peaks, due to fans and other rotating mechanical equipment, enter through acoustic and mechanical coupling mechanisms. Improvements in acoustic shielding and in the optical layouts to reduce acoustic sensitivity are planned to address these peaks.

The signal that is analyzed to search for gravitational wave signals is obtained from the photodiode at the antisymmetric port. Since this is the error signal for the differential arm length, the effect of the feedback loop gain must be measured and compensated for. The absolute scale for strain is established using the laser wavelength, measuring the mirror drive signal required to move through a given number of interference fringes. The frequency response of the detector is determined via swept-sine excitations of the end mirrors made periodically through the run.

One of the difficulties with the S1 data was that drifts in the alignment (because the wavefront sensing portion of the alignment control system was not fully operational) caused changes in the coupling of light into the interferometer. This shows up directly in changes in the optical gain of the interferometers (e.g., watts per meter at the antisymmetric port), and in changes of the overall gain of the servo system holding the interferometers in lock. The first effect is an overall scaling in the signal, and was typically $10 \%$ or less in S1. However, the change in servo gain gives a frequency-dependent correction to the calibration function, which can be several times larger than the overall scale change, particularly near the unity gain point of the differential arm servo (between 150 and $200 \mathrm{~Hz}$ ). To compensate for this problem, the length of one of the arms was modulated sinusoidally at two frequencies with known amplitudes using the actuators on one of the end test masses. By monitoring the size of the resulting signals in the antisymmetric port photodiode signal, the optical gain of the interferometer could be tracked on a minute-by-minute basis, and corrections for the drift applied. The calibration procedure and results are described in more detail in reference [42]; the overall statistical error is about $\pm 10 \%$.

Other measures of performance also showed non-stationarity. The band-limited $\mathrm{rms}$ in the antisymmetric port photodiode sometimes showed degradation during a locked section as the interferometer drifted away from the initial alignment. "Glitch" rates included variations of factors of 3100 between locked sections. This non-stationary behavior affects some searches more than others, but should improve as the detectors approach full operation.

\subsection{GEO 600}

The GEO 600 sensitivity for the S1 run are shown in Fig. 9. The duty cycle was $98 \%$ (see Table 3) and the longest continuous stretch of data is $121 \mathrm{~h}$. During the S1 run GEO 600 was operating in the power-recycled Michelson interferometer configuration with a reduced power recycling gain and reduced input laser power. The laser power injected into the first mode cleaner was attenuated to $2 \mathrm{~W}$. The overall optical transmission of the mode cleaners, phase modulators and isolators is $52 \%$ which leaves approximately $1 \mathrm{~W}$ of laser light being injected into the interferometer. The power buildup in the power recycling cavity was 300 which was limited by the $1.3 \%$ transmission of the power recycling mirror installed during S1. The signal recycling mirror was not installed and test mirrors suspended in wire slings were used for the beamsplitter and the inboard mirrors of the folded interferometer arms.

Due to the automatic alignment system [11] the longest duration without manual alignment of the mode cleaners was more than one year prior to S1 and no manual alignment of the mode cleaners was undertaken during the S1 run. Operator alignment of the power recycling cavity and the Michelson interferometer was needed only a few 


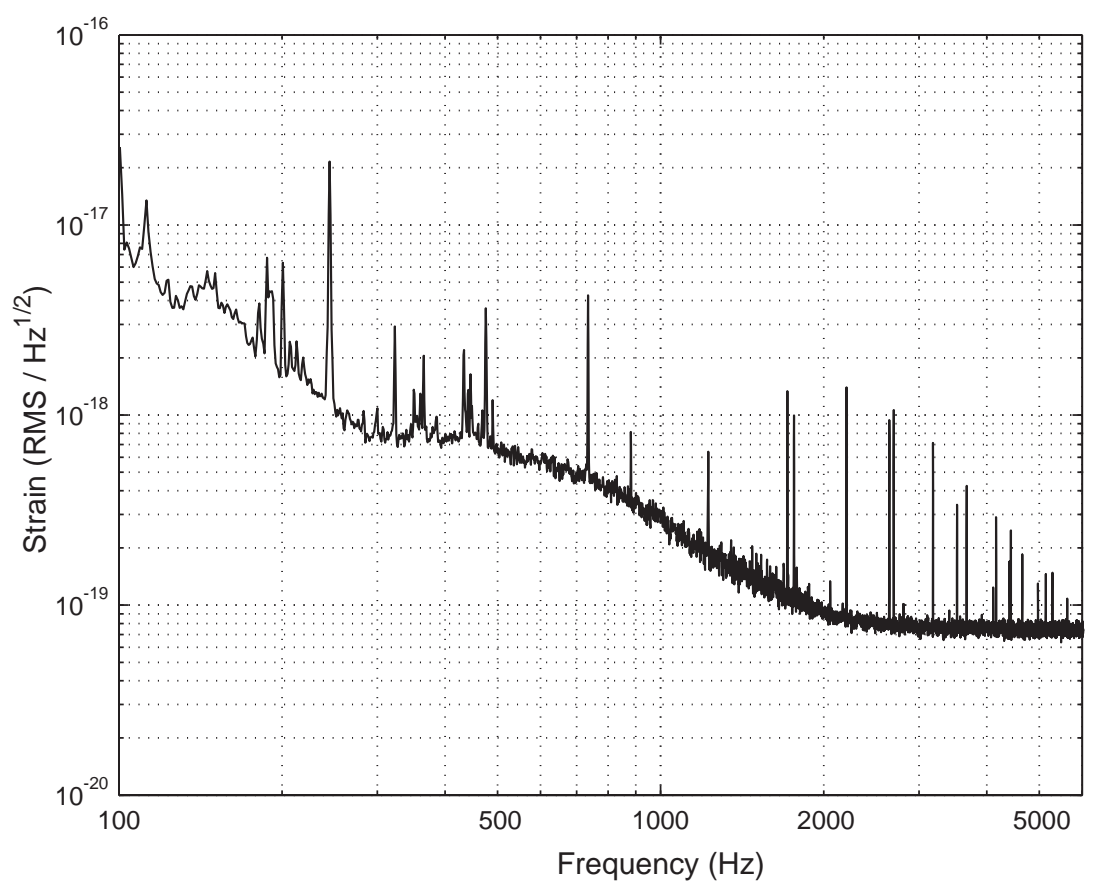

Fig. 9. The GEO 600 sensitivity curve for S1.

times after major seismic disturbances. An automatic lock acquisition process was initiated whenever any of the cavities or the Michelson interferometer lost lock. This system and the automatic alignment system were very stable and reliable and no operator presence at the detector was required at night.

Calibration of GEO 600 during the S1 run was achieved by imposing known forces on two of the mirrors using electrostatic drives that were fitted to provide differential control of the interferometer. In the final GEO 600 configuration, radiation pressure from a modulated laser beam which will be aligned onto and reflected by one of the end mirrors will introduce calibration lines with known amplitude into the output signal of the detector. The spectrum of the applied calibration force consisted of a line at $244 \mathrm{~Hz}$ and its odd harmonics, generated by suitable filtering from a square wave. The signal generator used to produce the series was phase-locked to the GPS stabilized clock to which the data acquisition system was also synchronized. The electrostatic actuators were calibrated with respect to electro-magnetic actuators one stage higher up on the same suspension chains. These were in turn calibrated by applying forces large enough to enable simple fringe counting. The actuators were found to be adequately linear to allow this calibration method to succeed with good reliability. The calibrated gravitational wave channel was generated by measuring the amplitudes of the calibration peaks in $1 \mathrm{~s}$ frames of data. These measurements were used to determine the unknown calibration factors (essentially the overall optical transfer function magnitude and the gain of the relevant control loops) by fitting the data to a model based on previously measured transfer functions of the electro-mechanical control system. The calibration coefficients were smoothed over periods of $1 \mathrm{~min}$. Suitable time domain digital filters were generated to produce a calibrated gravitational wave channel. The overall calibration uncertainty was about $4 \%$ for signal frequencies above $200 \mathrm{~Hz}$ and $6 \%$ between $50 \mathrm{~Hz}$ and $200 \mathrm{~Hz}$. Further detail of this process can be found in Ref. [43]. 


\section{Summary/future}

The $\mathrm{S} 1$ science run is an important milestone for LIGO and GEO, providing the first data for scientific analysis for two of the newest generation of gravitational wave interferometer observatories. Even though the detectors were operated in a preliminary configuration with many features not implemented, the data were relatively well behaved, and the sensitivity great enough to improve on prior observations with broadband gravitational wave detectors.

Several types of analysis have been recently completed using the data from the S1 run. These include:

- a search for the inspiral signal from binary neutron star mergers [44]

- a search for continuous waves from a rapidly rotating pulsar $(\mathrm{J} 1939+2134)[45]$

- a search for short bursts of gravitational waves from unknown sources [46]

- a search for a stochastic background of gravitational waves of cosmological origin [47]

In all cases, the sensitivity for $\mathrm{S} 1$ was not expected to be sufficient to make a positive detection, so the emphasis in these analyses is to develop techniques for searching for gravitational waves, to confront the problems of dealing with real data with its deviations from the usual assumptions of gaussianity and stationarity, and to set improved upper limits on the flux of gravitational waves incident on the Earth.

Continued rapid improvements are expected in both the LIGO and GEO detectors. Immediately after the S1 run, GEO commenced the installation and commissioning of the complete optical configuration by adding the signal recycling mirror. LIGO has continued to complete the control system and tuning, leading to more than a factor 10 improvement in the sensitivity. New science data runs are taking place to collect data with this better performance. There will be a smooth transition from the present epoch, where commissioning dominates, to the goal of effectively continuous astrophysical data collection, as the instruments approach their goal sensitivity. The data are already interesting in terms of constrain- ing astrophysical models from upper limits, and significant improvements in the near future will make even better upper limits possible, along with the increased potential of directly detecting gravitational waves of astrophysical origin.

\section{Acknowledgements}

The authors gratefully acknowledge the support of the United States National Science Foundation for the construction and operation of the LIGO Laboratory and the Particle Physics and Astronomy Research Council of the United Kingdom, the Max-Planck-Society and the State of Niedersachsen/Germany for support of the construction and operation of the GEO600 detector. The authors also gratefully acknowledge the support of their research by these agencies and by the Australian Research Council, the Natural Sciences and Engineering Research Council of Canada, the Council of Scientific and Industrial Research of India, the Department of Science and Technology of India, the Spanish Ministerio de Ciencia y Tecnologia, the John Simon Guggenheim Foundation, the David and Lucile Packard Foundation, the Research Corporation, and the Alfred P. Sloan Foundation. This paper has been assigned LIGO Laboratory document number LIGO-P030024.

\section{References}

[1] M. Ando and the TAMA collaboration, Class. Quantum Grav. 19 (2002) 1409.

[2] F. Acernese, et al., Class. Quantum Grav. 19 (2002) 1421.

[3] K. Danzmann, GEO 600-A 600-m Laser Interferometric Gravitational Wave Antenna, in: E. Coccia, G. Pizella, F. Ronga (Eds.), First Edoardo Amaldi Conference on gravitational wave experiments, World Scientific, Singapore, 1995, pp. 100-111.

[4] B. Willke, et al., Class. Quantum Grav. 19 (2002) 1377.

[5] A. Abramovici, et al., Science 256 (1992) 325.

[6] B. Barish, R. Weiss, Phys. Today 52 (1999) 44.

[7] B.J. Meers, Phys. Rev. D 38 (1988) 2317.

[8] G. Heinzel, et al., Phys. Rev. Lett. 81 (1998) 5493.

[9] M.V. Plissi, et al., Rev. Sci. Instr. 71 (2000) 2539.

[10] S. Gossler, et al., Rev. Sci. Instr. 74 (2003) 3787.

[11] H. Grote, et al., The automatic alignment system of GEO 600, Proceedings of the Fourth Edoardo Amaldi on 
Gravitational Waves, Class. Quantum Grav. 19 (2002) 1849.

[12] A. Freise, et al., The automatic alignment system of GEO 600, Proceedings of the Fourth Edoardo Amaldi on Gravitational Waves, Class. Quantum Grav. 19 (2002) 1389.

[13] L. Buboltz, et al., LIGO beam tube module design, fabrication \& installation, Proceedings of the Ninth International Conference of Pressure Vessel Technology, Vol. 1, 2000, p. 797.

[14] W.A. Carpenter, et al., J. Vac. Sci. Technol. A 18 (2000) 1794.

[15] W.E. Althouse, et al., Rev. Sci. Instr. 72 (2001) 3086.

[16] D. Li, D. Coyne, J. Camp, Appl. Opt. 38 (1999) 5378.

[17] R. Savage, P. King, S. Seel, Laser Phys. 8 (1998) 679.

[18] R.W.P. Drever, et al., Appl. Phys. B 31 (1983) 97.

[19] S. Whitcomb, et al., Optics development for LIGO, in: K. Tsubono, M.-K. Fujimoto, K. Kuroda (Eds.), Proceedings of the TAMA International Workshop on Gravitational Wave Detection, November 11-12, 1996, Saitama, Japan, Universal Academy Press, Inc., Tokyo, 1997, pp. 229-239.

[20] C.J. Walsh, A.J. Leistner, J. Seckold, B.F. Oreb, D.I. Farrant, Appl. Opt. 38 (1999) 2870.

[21] P. Saulson, Fundamentals of Interferometric Gravitational Wave Detectors, World Scientific, Singapore, 1994, ISBN 981-02-1820-6.

[22] J. Heefner, R. Bork, The LIGO suspended optic digital control system, in: H. Shoaee (Ed.), Proceedings of the Eighth International Conference on Accelerator and Large Experimental Physics Control Systems (ICALEPCS 2001) eConf C011127, WEAP059, 2001 [arXiv:physics/0110071].

[23] Yu. Levin, Phys. Rev. D 57 (1998) 659.

[24] J. Giaime, P. Saha, D. Shoemaker, L. Sievers, Rev. Sci. Instr. 67 (1996) 208.

[25] J.A. Giaime, et al., Rev. Sci. Instr. 74 (2003) 218.

[26] M.W. Regehr, F.J. Raab, S.E. Whitcomb, Opt. Lett. 20 (1995) 1507.

[27] P. Fritschel, et al., Appl. Opt. 40 (2001) 4988.

[28] M. Evans, et al., Opt. Lett. 27 (2002) 598.

[29] Y. Hefetz, N. Mavalvala, D. Sigg, J. Opt. Soc. Am. B 14 (1997) 1597.

[30] P. Fritschel, et al., Appl. Opt. 37 (1998) 6734.

[31] B. Bochner, Y. Hefetz, A grid-based simulation program for gravitational wave interferometers with realistically imperfect optics, Phys. Rev. Lett. 68 (8) (2003).

[32] H. Yamamoto, et al., End to End Simulation program for gravitational-wave detectors, in: S. Kawamura, N. Mio (Eds.), Gravitational Wave Detection II, Frontiers Science Series No. 32, 2000, pp. 331-336.

[33] EPICS home page: http://www.aps.anl.gov/epics/index.php
[34] D. Sigg, R. Bork, J. Zweizig, Detector characterization and global diagnostics system of the Laser Interferometer Gravitational-Wave Observatory (LIGO), Proceedings of the Ninth Marcel Grossman Conference, July 2-8, 2000, Rome, Italy, 2001.

[35] S. Anderson, et al., The LIGO data analysis system, in: J. Tran Thanh Van, J. Dumarchez, S. Reynaud, C. Salomon, S. Thorsett, J.Y. Vinet (Eds.), Gravitational Waves and Experimental Gravity: Proceedings of the XXXIVth Recontres de Moriond, January 23-30, 1999, Les Arcs, France, World Publishers, Hanoi, 1999, p. 243.

[36] J.K. Blackburn, Laser Interferometer Gravitational Wave Observatory (LIGO) data analysis system, Fourth MultiConference on Circuits, Systems, Communications \& Computers, 2000.

[37] E. Deelman, et al., High Performance Distributed Comput. HPDC-11 (2002) 225.

[38] G. Cagnoli, et al., Phys. Rev. Lett. 85 (2000) 2442.

[39] S. Rowan, S.M. Twyford, J. Hough, D-H. Gwo, R. Route, Phys. Lett. A 246 (1998) 471.

[40] I. Zawischa, et al., The GEO 600 laser system, Proceedings of the Fourth Edoardo Amaldi on Gravitational Waves, Class. Quantum Grav. 19 (2002) 1775.

[41] M.M. Casey, H. Ward, D.I. Robertson, Rev. Sci. Instr. 71 (10) (2000) 3910.

[42] R. Adhikari, G. Gonzalez, M. Landry, B. O'Reilly, Calibration of the LIGO detectors for the First Scientific Run, in: Proceedings of the Seventh Gravitational Wave Data Analysis Workshop, Kizu-cho, Kyoto, Japan, 17-19 December 2002, Class. Quant. Grav., 2003, to appear.

[43] M. Hewitson et al., Calibration of GEO 600 for the S1 science run, Class. Quantum Grav., 2003, to appear.

[44] LIGO Scientific Collaboration, Analysis of LIGO data for gravitational waves from binary neutron stars, Phys. Rev. D, 2003, submitted.

[45] LIGO Scientific Collaboration, Setting upper limits on the strength of periodic gravitational waves using the first science data from the GEO600 and LIGO detectors, Phys. Rev. D, 2003, submitted.

[46] LIGO Scientific Collaboration, First upper limits on gravitational wave bursts from LIGO, Phys. Rev. D, 2003, to be submitted.

[47] LIGO Scientific Collaboration, Analysis of First LIGO Science data for stochastic gravitational waves, Phys. Rev. D, 2003, to be submitted.

[48] A.Yu. Ageev, et al., Phys. Lett. A 227 (1997) 159.

[49] A.Yu. Ageev, et al., Phys. Lett. A 246 (1998) 479.

[50] S. Rowan, et al., Phys. Lett. A 233 (1997) 303.

[51] V.B. Braginsky, et al., Physics Doklady 40 (1995) 564.

[52] V.B. Braginsky, et al., Phys. Lett. A 218 (1996) 164. 